\title{
Low-concentration sevoflurane inhalation in treating MK801-induced schizophrenia like disease in mice and a feasibility study of schizophrenia patients
}

Tianyun Zhao

Guangzhou Women and Children's Medical Center

\section{Shi Ziwen}

Guangzhou Women and Children's Medical Center

Nongxi Ling

The Third People's Hospital of Xinhui District

\section{Ying Xu}

MOE Joint International Research Laboratory of CNS Regeneration, Guangdong-Hong Kong-Macau Institute of CNS Regeneration (GHMICR), Jinan University

Jingwen Qin

Guangzhou Women and Children's Medical Center

\section{Quancai Zhou}

The Third People's Hospital of Xinhui District

\section{Lingzhi Wu}

Imperial College London, Chelsea and Westminster hospital

\section{Yuansheng Wang}

Jiangmen Central Hospital

\section{Wei Wei}

Guangzhou Women and Children's Medical Center

Chuansong Lin

The Third People's Hospital of Xinhui District

Daqing Ma ( $\sim$ d.ma@imperial.ac.uk)

Imperial College London, Chelsea and Westminster hospital https://orcid.org/0000-0002-0688-2097

\section{Xingrong Song}

Guangzhou Women and Children's Medical Center

\section{Article}

Keywords: sevoflurane, schizophrenia, GABAARs, sociability, E/I balance

Posted Date: August 28th, 2020 
DOI: https://doi.org/10.21203/rs.3.rs-49575/v1

License: (c) (i) This work is licensed under a Creative Commons Attribution 4.0 International License. Read Full License 


\section{Abstract}

GABAergic deficits have been considered to associate with the pathophysiology of schizophrenia and hence GABA receptor subtype $A\left(G A B A_{A} R s\right)$ modulators may have therapeutic values for schizophrenia. Sevoflurane, a commonly used volatile anesthetic, enhances GABAergic neurotransmission through the $G_{A B A} R$. The present study aims to investigate the therapeutic effectiveness of low-concentration sevoflurane in MK801-induced schizophrenia-like mice and amongst schizophrenia patients in a single arm trial. Three weeks after administration of MK801 $(0.5 \mathrm{mg} / \mathrm{kg}$, i.p. twice a day) for five days, mice were exposed to $1 \%$ sevoflurane for $1 \mathrm{hr} /$ day for 5 days. One week after treatment, they were subjected to behavioral tests, and then sacrificed for immunohistochemical stain, western blot assay and electrophysiology recordings in the prefrontal cortex. Ten schizophrenia patients received 5-hr sevoflurane (0.5-1.2\%) for 6 days, and were assessed with the PANSS and the BPRS-18 in the 1 st and 2 nd week after the treatments. MK801 induced hypolocomotion and social deficits, downregulated the expression of NMDARs subunits, and postsynaptic density protein 95 (PSD95), reduced parvalbumin- and GAD67positive neurons, and changed the amplitude and frequency of mEPSC and mIPSC and evenly increased the excitation/inhibition (E/I) ratio. All these changes induced by MK-801 were attenuated by sevoflurane administration. Schizophrenia symptoms assessed with the scales were significantly improved in the 1st and 2nd week after treatments. Low-concentration sevoflurane inhalation effectively reversed MK801induced schizophrenia-like disease in mice and alleviated schizophrenia patients' symptoms. Our work suggested that sevoflurane may be a valuable therapeutic strategy for treating schizophrenia patients.

\section{Introduction}

Schizophrenia is a chronic and devastating psychiatric disorder. It is clinically characterized by both positive (i.e., hallucinations, delusions, abnormal motor behavior) and negative symptoms (i.e., avolition and sociality) as well as cognitive deficits, and affects up to $1 \%$ population world-wide ${ }^{1}$. It is ranked among the top 25 leading causes of disability globally and inflicts substantial social, healthcare and economic burdens ${ }^{2}$. Antipsychotics are the standard medication for schizophrenia and, indeed, dopamine or/and serotonin modulating antipsychotics remain the primary approved treatment for schizophrenia ${ }^{3}$. However, the majority of these antipsychotics are mainly effective for positive symptoms and leave most patients with negative symptoms and their cognitive dysfunction relatively untreated, and thus, patients have poor lifelong quality of lives ${ }^{4,5}$. In addition, due to their different affinity towards various synaptic receptors, these drugs are associated with nonadherence owing to their side effects including extrapyramidal (EP), cardiotoxicity and abnormal metabolic syndrome (hyperglycemia, hyperlipidemia, weight gain) make them not ideally for long term use ${ }^{6,7}$. The acute phase such as the first episode, or an acute exacerbation of schizophrenia is a pivotal phase because optimal treatment for the episode might improve the long-term prognosis ${ }^{8}$. Early diagnosis and, prompt and noncoercive interventions to relieve the symptoms during acute episode are critical to prevent aggressive behavior and symptomatic escalation. Indeed, delayed treatments and poor treatment compliance are considered to be significant barriers for the effective management of psychiatric conditions and the establishment of therapeutic 
alliance between patients and healthcare providers. The consensus for schizophrenia treatment is that any ideal medication should have the properties of rapid onset of action, calmness without sedation, easy to administer, non-invasive, non-traumatic/non-coercive, with good safety profile and favorable tolerability ${ }^{9}$.

$Y$-aminobutyric acid type $A$ receptors $\left(G A B A_{A} R\right)$ are a family of ligand-gated ion channels essential for the regulation of synaptic inhibition in the brain ${ }^{10}$. Aberrant $\mathrm{GABA}_{A} \mathrm{R}$ activities or dysregulation thereof are associated with various neurodevelopmental disorders, neurodegenerative diseases and psychiatric diseases, including autism, Alzheimer's, depression and schizophrenia ${ }^{11,12}$. With growing studies on $\mathrm{Y}^{-}$ aminobutyric acid type $A\left(G A B A_{A}\right)$ receptors $\left(G A B A_{A} R\right)$ accessory proteins and the molecules modulating receptor signaling in the central nervous system, targeting $G A B A_{A} R$ and associated proteins might shed a light to novel therapeutic development for psychotic disorders. Sevoflurane, a commonly used volatile anesthetic for surgical anesthesia, is a potentiator of $\gamma$-aminobutyric acid type $A\left(G A B A_{A}\right)$ receptors and can induce rapid and well-controlled sedation ${ }^{13}$. Because of these advantageous properties, sevoflurane and other inhalational anesthetics are increasingly used as general sedatives for invasive diagnostic and therapeutic procedures, mechanical ventilation and management of agitation in intensive care unit. The concentration required for sedation is approximately one third ( 0.3 minimum alveolar concentration) of the concentration required for general anesthesia with less incidence of nausea and vomiting ${ }^{14}$. The present study aims to test our hypothesis that inhaling low concentration of sevoflurane attenuates the abnormal behaviors and reverses the changes to $\mathrm{GABA}_{A} \mathrm{R}$ associated proteins in a mice model of MK801induced schizophrenia. We also evaluated the therapeutic benefit of sevoflurane at low concentration in a preliminary group of schizophrenia patients.

\section{Results}

\section{MK801 caused a persistent body weight reduction in mice}

Most studies have consistently described that MK801 administration results in decreased weight compared to controls in rodents, and this weight retardation can persist into adulthood. We therefore measured body weight on a daily basis during MK801 treatment and once a week thereafter till the P32. Two-way ANONA with repeated measures revealed a significant effect of MK801-treatment (Fig. 1, F $(1,87)=76.87, p<0.01)$. Subsequent post hoc tests showed persistent lower body weight from P11 to P31 in MK801-treated mice compared with controls (Fig. 1, p $<0.01$ at P11, and $p<0.01$ at the P17, P24 and P31).

\section{Sevoflurane treatment rescued Mk801-induced hypoactivity in the OFT}

OFT was performed 7 days after sevoflurane exposure to investigate the spontaneous locomotor activity, with the example of trials in each group showed in Fig. 2A-C. One-way ANOVA showed a significant effect 
on total distance between three groups (Fig. 2D, F $(2,51)=32.92, p<0.001)$. Bonferroni's post hoc tests showed that administration of MK801 significantly decreased the total distance compared with that in the control group (Fig. 2D, p < 0.001). Sevoflurane exposure (MK801 + Sev) significantly increased the total distance compared with that in the MK801 group (Fig. 2D, p < 0.001). There was no significantly difference between the control and the MK801 + Sev group (Fig. 2D, $p=0.090$ ). Similarly, One-way ANOVA showed a significant effect on the frequency of crossing squares between groups (Fig. 2E, F $(2,51)=$ $22.35, p<0.001)$. Bonferroni's post hoc tests showed that the MK801 group crossed fewer squares than the control group (Fig. 2E, $p<0.001$ ), while the MK801 + Sev mice displayed significantly higher frequency of crossing squares compared with the MK801 group (Fig. $2 E, p<0.001$ ).

\section{Sevoflurane treatment restored MK801-induced social interaction defects}

During the social approach phase, the test mice in three groups explored freely in each chamber, as the example of recording trials and density map shown in Fig. 3A-B (Ctrl: left; MK801: middle; MK801 + Sev: right, respectively). There was no difference in the time exploring the left chamber with an empty black wire cup inside (Fig. 3C), while the times exploring the center chamber (Fig. 3D) and the right chamber with a novel mouse inside the wire cup (Fig. 3E) were significantly different. Bonferroni's post hoc tests showed that MK801-treated mice spent more time lingering in the center chamber and less time in the right chamber than mice in the control and MK801 + Sev groups (Fig. 3C-D). The time sniffing the novel animal revealed significant difference between three groups (Fig. $3 F, F(2,51)=6.75, p=0.003$ ). The MK801-treated mice spent less time sniffing the novel animal than the control group (Fig. 3F, $p=0.003$ ). The MK801 + Sev group mice spent more time sniffing the novel animal than the MK801 group mice (Fig. $3 F, p=0.028$ ), while there was no significant difference between the MK801 + Sev and control groups (Fig. 3F, $\mathrm{p}=0.659$ ).

\section{Sevoflurane reversed MK801-induced aberrant NMDARs composition in the mPFC}

The expression of NMDARs subunits including NR1, NR2A and NR2B within the prefrontal cortex were determined by western blot assay. There were no significant differences in the expression of NR1 in the three groups (Fig. 4A, B, Figure $F(2,15)=0.52, p=0.602$ ). However, NR2A was differentially expressed between the three groups (Fig. 4C, D, F $(2,15)=14.21, p<0.001$ ). Bonferroni's post hoc test showed that the expression of NR2A was significantly decreased in the MK801 group compared to that of control group (Fig. 4D, $p<0.001$ ), while the expression of NR2A in the MK801 + Sev group was higher than that of MK801 group (Fig. 4D, $p=0.021$ ). Similarly, NR2B expression was different amongst three groups (Fig. 4E, F, F $(2,15)=15.58, p<0.001)$. Bonferroni's post hoc tests showed that the expression of NR2B was significantly decreased in the MK801 group compared to that of control group (Fig. 4D, p<0.001), while the expression of NR2B in the MK801 + Sev group was significantly increased, as compared to that of MK801 group (Fig. 4F, p = 0.019). In addition, there was a significant difference of the expression of 
PSD95 among three groups (Fig. $4 \mathrm{H}, \mathrm{F}(2,15)=9.44, p=0.002)$. Bonferroni's post hoc tests showed that PSD95 expression was significantly decreased in the MK801 group compared to that of the control group (Fig. 4F, $p=0.002$ ), while the expression of PSD95 in the MK801 + Sev group was significantly increased, as compared to that of the MK801 group (Fig. $4 F, p=0.022$ ).

\section{Sevoflurane reversed MK801-induced alterations of GABAergic neuronal transmission and ErBb4-NRG1 signal pathway in the mPFC}

There was a significant difference in the number of PV positive interneurons in the mPFC as indicated by blue area in Fig. 5A (Fig. 5B-E, F $(2,18)=7.26, p=0.005$ ). Bonferroni's post hoc tests showed that the density of PV interneurons was significantly decreased in the MK801 group compared to that of control group (Fig. 5B, $p=0.006$ ), while the density of PV interneurons in the MK801 + Sev group was significantly increased, as compared to that of MK801 group (Fig. 5B, $p=0.023$ ). Similarly, there was a significant difference in the density of GAD67 cells between the three groups (Fig. 6D, F $(2,18)=13.51, p$ $<0.001)$. Bonferroni's post hoc tests showed that the density of GAD67 cells was significantly decreased in the MK801 group compared to that of control group (Fig. 6D, p < 0.001), while the density of GAD67 cells in the MK801 + Sev group was significantly increased, as compared to that of MK801 group (Fig. 6D, $p=0.003)$. However, no differences in the expression of $G_{A B A_{A}}$ receptor subunits were observed, including $\mathrm{a} 1, \beta 2$ and the anchoring protein gephyrin (Fig. 7A, B, GABA $A$ a1, $F(2,15)=0.59, p=0.568$; Fig. 7C, $\mathrm{D}, \mathrm{GABA}_{\mathrm{A}} \beta 2, \mathrm{~F}(2,15)=0.25, p=0.784$, and Fig. $7 \mathrm{E}, \mathrm{F}$, gephyrin, $\mathrm{F}(2,15)=0.71, p=0.507$, respectively). The ErBb4-NRG1 signal pathway was explored to see whether it is the underlying mechanism sevoflurane acts through to ameliorate schizophrenia. One-way ANOVA showed that there was a significant difference in the expression of ErBb4 in the three groups (Fig. $7 \mathrm{H}, \mathrm{F}(2,15)=10.86, p=$ 0.001). Bonferroni's post hoc tests showed that the expression of ErBb4 was significantly decreased in the MK801 group compared to that of control group (Fig. 7G, $\mathrm{H}, \mathrm{p}=0.002$ ), while the expression of ErBb4 in the MK801 + Sev group was significantly increased, as compared to that of MK801 group (Fig. 7H, $p=$ 0.004). Similarly, One-way ANOVA showed that there was a significant difference in the expression of NRG1 between the three groups (Fig. 7I, J, F $(2,15)=5.22, p=0.019$ ). Bonferroni's post hoc test showed that the expression of NRG1 was significantly decreased in the MK801 group compared to that of control group (Fig. 7J, $p=0.045$ ), while the expression of NRG1 in the MK801 + Sev group was significantly increased, as compared to that of MK801 group (Fig. 7J, $p=0.027$ ).

\section{Sevoflurane reversed MK801-induced alterations of electrophysiological profiles and E/I balance in the MPFC}

To identify whether sevoflurane treatment can reverse the MK801-induced alterations to electrophysiological characteristics of pyramidal neurons in the laminar II/III of the mPFC, whole-cell recordings were performed 2 weeks after sevoflurane treatment at P49. The excitatory postsynaptic currents (mEPSCs) recorded in the mPFC pyramidal neurons of the control (Fig. 8A), MK801 (Fig. 8B) and 
MK801 + Sev (Fig. 8C) showed that there was a significant difference in the mean amplitude of mEPSCs in the three groups (Fig. 8G, p < 0.001). Dunn's multiple comparisons tests showed that the mean amplitude was significantly decreased in the MK801 group compared to that of control group (Fig. 8G, p $<0.001$ ), while the mean amplitude in the MK801 + Sev group was significantly increased, as compared to that of MK801 group (Fig. 8G, p < 0.001). Unexpectedly, in the MK801 group, the frequency cumulative distribution plot showed a distinct leftward shift, with a significant increase in the mean frequency of miniature excitatory postsynaptic currents (mEPSCs) compared to the control (Fig. 8H, p < 0.001) analyzed with Dunn's multiple comparisons tests, while the mean frequency of mEPSCs was reversed in the MK801 + Sev group, as compared to the MK801 group (Fig. 8H, $p=0.005$ ). In addition, analysis of the miniature inhibitory postsynaptic currents (mIPSCs) recorded in the mPFC pyramidal neurons of the control (Fig. 8C), MK801 (Fig. 8D) and MK801 + Sev (Fig. 8E) using Kruskal-Wallis test revealed significant difference in the mean amplitude of mIPSCs between the three groups (Fig. 8I, F $(2,68)=$ $12.03, p<0.001)$. Dunn's multiple comparisons tests showed that the mean amplitude of mIPSCs was significantly decreased in the MK801 group compared to that of control group (Fig. 8l, $p<0.001$ ), while the mean amplitude in the MK801 + Sev group was significantly increased, as compared to that of MK801 group (Fig. 8l, $p=0.005$ ). However, there was no significant difference in the mean frequency of mIPSCs between groups (Fig. $81, p=0.518$ ). These alterations of mEPSCs and mIPSCs may change the glutamatergic and GABAergic synaptic neurotransmission of the pyramidal neurons, resulting in $\mathrm{E} / \mathrm{I}$ imbalance in the mPFC. Indeed, as shown in Fig. 8M, the E/I ratio (mEPSC/mIPSC amplitude of the same pyramidal neuron) was significantly different between groups (Fig. 8M, $F(2,52)=19.63, p<0.001)$. Dunn's multiple comparisons tests showed that the E/I ratio of in the MK801 group was higher than that of control group (Fig. 8M, p < 0.001), which suggested a toward of hyperexcitation. The MK801 + Sev group was significantly decreased E/I ratio to the level of MK801 group (Fig. 8M, $p<0.001$ ).

\section{Clinical trial results}

Nine out of ten enrolled patients (seven male and three female) completed all 6 trial treatments and because of postural hypotension after treatment, 1 patient was stopped to participate after received one treatment (a male patient-no 3 in Fig. 9). Data from all patients were included in the final data analysis. The clinical and demographic characteristics for the patients and their baseline PANSS and BPRS scores are shown in appendix D: Table 2.

\section{Primary efficacy}

Eight patients achieved 30\% decrease in PANSS total score from baseline to end of week 2 following sevoflurane treatment (appendix E: Table 3). A total 6 patients out of 10 patients (full analysis set) achieved $30 \%$ decrease in PANSS total score from baseline to end of week 1 following sevoflurane treatment. The BPRS-18 total score revealed a rapid decrease from the baseline to week 1 and week 2 . The BPRS-18 total score significantly improved over time with a mean $( \pm S D)$ change from baseline of 
$-9.7( \pm 10.60)$ at the end of week 1 , and to $-30.9( \pm 19.51)$ at the end of week 2 (Fig. 9). One patient with disease history of 16 years received one electroconvulsive therapy given that the clinical symptoms didn't improve after three times of treatments.

\section{Safety}

No abnormal physiological parameters and no sevoflurane-related side effects were found and patients tolerated the treatment well.

\section{Discussion}

The present study aims to investigate the potential therapeutic effects of low-concentration sevoflurane inhalation on behavior deficits and perturbations to glutamatergic and GABAergic neurotransmission in a MK801-induced mice model that mimics negative features of schizophrenia. Our findings were as follows: i) Mice treated with MK801 at $0.5 \mathrm{mg} / \mathrm{kg}$ (twice/day for 5 days) displayed hypolocomotion and impaired sociability; ii) Low-concentration of sevoflurane reversed these behavioral deficits; iii) Lowconcentration of sevoflurane attenuated neurochemical and electrophysiological alterations, and restored NMDA glutamatergic and GABAergic neurotransmission. In view of the pharmacological advantages of sevoflurane including rapid onset, relative non-toxicity, noninvasive route of administration and lesser side effects than currently available antipsychotics, our findings indicate that sevoflurane may be a potential therapy for treating an acute episode and negative symptoms of psychotic disorders.

Previous studies suggest the glutamatergic and GABAergic neurons and their neurotransmitter systems play crucial roles in the pathophysiology of schizophrenia ${ }^{12}$; but, currently, there are no relevant new medications available for clinical use. $\mathrm{GABA}_{A}$ receptors are heteropentameric structures assembled from a large family of subunits including two a $(a 1-6)$, two $\beta(\beta 1-3)$, and either one $\gamma(\gamma 1-3)$ or one $\delta$ subunit 15 . The majority of $\mathrm{GABA}_{A}$ receptors are comprised of $\alpha 1, \beta 2$, and $\gamma 2$ subunits located at GABAergic synapses via direct association with key scaffolding protein gephyrin which is essential for phasic $\mathrm{GABA}_{A} \mathrm{R}$ signaling ${ }^{16}$. Among the major neurotransmitter-gated ion channels or ionotrophic receptors, $G_{A B A_{A}}$ receptor is critical for the hypnosis action of general anesthetics. Volatile anesthetics (such as isoflurane, sevoflurane and desflurane) enhance the response of the $G A B A_{A} R s$ to $G A B A$, increase the duration of inhibitory response, and potentiate the duration of GABA-mediated synaptic inhibition ${ }^{17}$. In addition, there is 'direct activation' of $G A B A_{A} R$ by opening the anion channel at higher concentrations in the absence of $\mathrm{GABA}^{18}$. We found that 1 -hour $1 \%$ sevoflurane inhalation for 5 days reversed the decreases in locomotion and sociability induced by MK801. OFT was adopted to access the exploratory behaviors and emotional disorders including anxiety-like and depression-like behaviors ${ }^{19,}{ }^{20}$, whilst an automated three-chambered social approach task was used for evaluating sociability ${ }^{21}$. Our results are in agreement with studies showing that administration of MK801 to neonatal animals induced reduction in spontaneous locomotor activity in long-term which may reflect psychomotor retardation ${ }^{22,23,24}$. For 
example, a consecutive 5-day administration of MK801 in neonatal Balb/c mice (P7) produced a negative model of schizophrenia including hypoactivity in OFT 22.

Glutamate (Glu) and GABA, as the major excitatory and inhibitory neurotransmitter in the brain, respectively, are crucial in maintaining the normal brain function. Glutamate and GABA neurotransmitter systems play central roles in the pathophysiology of mood disorder. NMDAR antagonists, e.g., phencyclidine (PCP) and MK801, can induce symptoms of schizophrenia in normal human subjects 25,26 , 27. NMDARs, which predominantly localize at the excitatory postsynaptic membrane and interact with the postsynaptic density 95 (PSD95), are ionotropic glutamate receptors comprised of two conserved NR1 subunits and two NR2 and/or NR3 subunits. The heteromeric NMDARs provide a wide variety of wellorganized composition of subunits to engage in specific neuronal functions within different brain regions. Therefore, it is not surprising that disturbed composition, misplacement and abnormal trafficking of NMDAR subunits were found in pathological conditions, psychiatric diseases and developmental disorders ${ }^{28}$. NMDARs show rapid maturation with switching from NR2B- to NR2A-NMDARs during the early development in mice, and are prone to MK801-induced schizophrenia-like symptoms ${ }^{29,30,31}$. Indeed, in our study, sub-chronic administration of MK801 during rapid neurodevelopmental phase led to disturbed composition of NMDARs subunits, with downregulation of NR2A and NR2B subunits and the scaffolding protein PSD95, but NR1 subunit expression remained unchanged. A previous study found GluN1 and GluN2A downregulation in the prefrontal cortex of postmortem brain of patients with schizophrenia ${ }^{32}$. A few studies also reported NMDARs subunits alteration after MK801 systemic administration in rodents ${ }^{33,34}$. In addition, cell adhesion molecule neuregulin 1 (NRG1) and its neuronal tyrosine kinase receptor ErbB4 were widely expressed in the interneurons in schizophrenia subjects ${ }^{35}$. NRG1, NR2 subunits and ErbB4 share a common anchoring region on the postsynaptic sites and NRG1ERBB4 signaling can affect NMDAR and its function ${ }^{36}$. Indeed, we found the expressions of NRG1, ErbB4, NR2A, NR2B and PSD95 were significantly decreased following MK801 administration and lowconcentration sevoflurane reversed all these changes induced by MK-801.

Prefrontal cortex (PFC) plays a fundamental role in regulating multiple complex behaviors, including memory, cognitive, attention, social interaction and emotional regulation. The PV interneurons, the most common subtype of GABAergic neurons known for their fast-spiking phenotype, have a strong control over the excitability of pyramidal neurons by innervating the soma and proximal dendrites ${ }^{37}$. Therefore, PV interneurons are powerful regulators for maintaining optimal E/I balance within prefrontal local circuits for information processing. Previously, it has been demonstrated that disrupting E/I balance in the PFC via pharmacological, chemogenetic and optogenetic approaches induced a range of PFCdependent abnormalities associated with psychiatric diseases ${ }^{38}$. MK801 administration likely disrupts the E/I balance in pyramidal neurons in laminar II-III of the MPFC via the reduced GABAergic neurotransmission and hypofunctional NMDARs. Indeed, we found that both excitatory and inhibitory synaptic transmissions in the pyramidal neurons were impaired by MK801 administration, evidenced by the decreased amplitude of MEPSC and mIPSC, the increased frequency of $\mathrm{mEPSC}$ and as a result, the 
$\mathrm{E} / \mathrm{I}$ ratio was increased. Low-concentration of sevoflurane increased GABAergic neurotransmission and reversed these electrophysiological changes induced by MK801.

Our proof of concept study indicated that patients tolerated the treatment very well and symptoms were noticeably improved by the treatment. Most importantly, of these patients, five patients with disease history over 10 years, and whose symptoms that failed to respond to routine anti-schizophrenia medications, were improved with this novel therapy. The most likely antipsychotic action of sevoflurane maybe attributable to its ability to enhance GABAergic neurotransmission, the deficit of which underlies the pathophysiology of schizophrenia. However, other mechanisms may be also involved: 1) subanesthetic concentration (0.4 MAC) of sevoflurane increases regional cerebral blood flow ${ }^{39}$, and lowconcentration of sevoflurane promotes hippocampal neurogenesis 40,41 . 2) Beneficial interactions between GABAergic neurons and serotonergic neurons ${ }^{42}$. Dexmedetomidine, a highly selective and potent $a_{2}$-adrenergic receptor $\left(a_{2}-A R\right)$ agonist, is widely used for induction. In this trial, it was used for initial sedative induction and then sevoflurane can be delivered smoothly to participants. Whether the beneficial effects we found in our patients are likely to be due to the interactive effects of both sevoflurane and dexmedetomidine remain unknown and warrant further study.

In summary, present study further substantiated the finding that MK801 administration to neonatal mice could induce a negative model of schizophrenia 43,44, 45 . Specifically 22, 23, 31 , a consecutive 5-day administration protocol of MK801 in neonatal mice (P7) induced negative symptoms of schizophrenia including hypoactivity and less sociability. These abnormalities could be explained by changes to NMDA receptor subtypes and GABAergic neurotransmitter, and the consequential excitatory/inhibitory neurotransmission imbalance in the pyramidal neurons in laminar II-III of the PFC. All these changes can be reversed by sevoflurane administration. Furthermore, low-concentration of sevoflurane inhalation effectively reversed MK801-induced negative symptoms of schizophrenia. The good tolerance and effectiveness of sevoflurane in schizophrenia patients noted in the current study would encourage further large-scale clinical study to comprehensively evaluate the therapeutic values of sevoflurane for schizophrenia patients.

\section{Materials And Methods}

\section{1) Pre-clinical studies}

\section{Subjects and Drugs}

\section{Subjects}

All experimental procedures were performed in accordance with the guidelines of the Animal Experiment Ethics Committees of Timed-pregnant Balb/c mice were purchased from

They were housed in a temperature and humidity-controlled room $\left(23 \pm 1^{\circ} \mathrm{C}, 45-55 \%\right)$ of 12 -hour 
light/dark cycle (lights on at 08:00 a.m.) with free access to water and food. The day of birth was designated as the postnatal day $0(\mathrm{P} 0)$. On the P7, they (weighing 4-6 g) were randomly allocated into three groups: control group (Ctrl group), MK801 group, and MK801 + Sevoflurane group (MK801 + Sev group). At the time of weaning (P21), mice were separated from their mothers and housed in group of four to six per cage.

\section{Drugs administration}

MK801 (Ref. M107, St. Louis, MO, USA) was used to induce negative symptoms of schizophrenia as reported previously ${ }^{43}$. It was dissolved in $0.9 \%$ sterile saline. At the P7, pups received intraperitoneal (i.p.) injection of $0.5 \mathrm{mg} / \mathrm{kg}$ MK801 in the MK801 group and MK801 + Sev group or an equal volume of saline (Ctrol group) twice a day (at 10:00 am and 15:00 pm) for five consecutive days. Their body weights were measured every day during the treatment period and once/week since cessation of the treatments till the P31. During the P31-P35, the MK801 + Sev group mice were placed in a $25 \mathrm{~cm} \times 20 \mathrm{~cm} \times 15 \mathrm{~cm}$ plexiglass chamber, and $1 \%$ sevoflurane was delivered via inlet of the chamber via a sevoflurane vaporizer (Easy fill / Cagemount, R58S, ........................) at $1 \mathrm{~L} / \mathrm{min}$ in $30 \%$ oxygen balanced with nitrogen for $1 \mathrm{hr}$ (10:00 am to $11 \mathrm{am}$ ) for five consecutive days. The concentration of sevoflurane was monitored with a gas monitor (BeneView T8, Mindray Bio-Medical Electronics Co.Ltd., ..) via the outlet of the chamber. The MK801 and control groups received identical gases without sevoflurane under the identical setting for $1 \mathrm{hr}$ for 5 days. Open field test was performed at the P43-P45, and then three-chamber social test was conducted after two non-stimuli days at the P47. After behavioral tests (see below), they were sacrificed under terminated anesthesia and their brain samples were harvested for electrophysiological recordings, western blot and immunohistochemical analysis, respectively. The experimental timeline is presented in Appendix: Supplementary Fig. 1.

\section{Behavioral test}

In order to avoid possible behavioral testing biases, mice were tested in a random order and all trails were carried out in the day at the same time period (9:00-16:00). In addition, the apparatus was cleaned with $70 \%$ ethanol after each trail to eliminate any effects from olfactory perception.

\section{Open field test (OFT)}

Open field test was adopted for assessing spontaneous locomotor activity. Mice were gently placed in the center of an open-top apparatus with 25 equal squares $(50 \mathrm{~cm} \times 50 \mathrm{~cm} \times 40 \mathrm{~cm})$ and allowed to move freely for $15 \mathrm{~min}$. Distances and traces of 15 min movements were recorded with a video camera and analyzed using EthoVision XT 8.0 (Noldus, Wageningen, Netherlands), the total distance travelled and the number of squares crossed during recording period were calculated for further analysis.

\section{Three-chamber sociability test}

The three-chamber test was conducted to assess social interaction with time spent in a side chamber with a novel mouse in a wire cup versus time spent in a side chamber with an empty identical wire cup ${ }^{46}$. 
A rectangular plexiglass box $(60 \mathrm{~cm} \times 40 \mathrm{~cm} \times 20 \mathrm{~cm})$ without a top cover was divided into three chambers $(20 \mathrm{~cm} \times 40 \mathrm{~cm} \times 20 \mathrm{~cm}$ ) with two partitions. Between each chamber, there is a $5-\mathrm{cm}$ opening hole which can be closed or opened with a lever operated door. The middle chamber was empty, while the side chamber contained an empty wire cup or an identical wire cup with a novel sex- and weight-matched mouse in. The test was divided into two phases. In brief, after a ten-minute habituation period (phase 1), the test mouse was placed in the middle chamber and allowed free access to visit each chamber. For the social approach phase (phase 2), the mouse was placed in the central compartment and left to explore with either an empty black wire cup (in left side chamber) or a similar wire cup with a novel mouse inside (the right side chamber). The apparatus and wire cups were thoroughly cleaned with $70 \%$ ethanol between phases and after each trial. Behaviors were videotaped by an automated tracking software (TopScan/ObjectScan, Cleversystems, Leesburg, VA, USA). The time spent in each compartment, and the time explored (sniffing) within a $2 \mathrm{~cm}$ vicinity of the cup with a novel mouse in was calculated to assess sociability.

\section{Electrophysiological recording}

\section{Slice preparation}

Slices of prefrontal cortex were prepared as previously described ${ }^{47}$. In brief, coronal brain slices $(250 \mu \mathrm{m})$ containing the PFC were cut with a vibratome (VT120S, Leica Microsystems) in ice cold solution containing (in mM): 75 sucrose, $85 \mathrm{NaCl}, 2.5 \mathrm{KCl}, 1.25 \mathrm{NaH}_{2} \mathrm{PO}_{4}, 4 \mathrm{MgSO}_{4}, 0.5 \mathrm{CaCl}_{2}, 24 \mathrm{NaHCO}_{3}, 25 \mathrm{D}$ glucose saturated with $95 \% \mathrm{O}_{2} / 5 \% \mathrm{CO}_{2}$, and recovered in a chamber filled with artificial cerebrospinal fluid (ACSF) (in mM: $119 \mathrm{NaCl}, 2.5 \mathrm{KCl}, 1.25 \mathrm{NaH}_{2} \mathrm{PO}_{4}, 2 \mathrm{MgCl}_{2}, 2 \mathrm{CaCl}_{2}, 26 \mathrm{NaHCO}_{3}, 10$ D-glucose) saturated with $95 \% \mathrm{O}_{2}$ and $5 \% \mathrm{CO}_{2}$ at $32 \circ \mathrm{C}$ before recording.

\section{Whole-cell recording}

After incubation, slices were transferred to a recording chamber where perfused at $2-3 \mathrm{ml} / \mathrm{min}$ with ACSF, saturated with $95 \% \mathrm{O}_{2}$ and $5 \% \mathrm{CO}_{2}$ and maintained at $32 \pm 2{ }^{\circ} \mathrm{C}$. The pyramidal neurons of PFC layer II/III were viewed with an Olympus microscope equipped with infrared DIC optics. The patch recording pipettes (4-6 M $\Omega$ ) were filled with a solution (in $\mathrm{mM}$ : 135 cesium methanesulfonate, $8 \mathrm{CsCl}, 0.25$ EGTA, 10 HEPES, $7 \mathrm{Na}_{2}$-phosphocreatine, $0.34 \mathrm{Na}_{3}$-GTP, $2.168 \mathrm{Mg}$-ATP, $\mathrm{pH}=7.2-7.3$, with $\mathrm{CsOH}$ ) and access resistance of recorded cells was less than $30 \mathrm{M} \Omega$. mEPSCs /mIPSCs were recorded at holding potential of $-70 \mathrm{mV} / 0 \mathrm{mV}$ in ACSF supplemented with $1 \mu \mathrm{M}$ tetrodotoxin. Only cells with membrane potentials lesser than $-65 \mathrm{mV}$ and series resistance below $25 \mathrm{M} \Omega$ were included for further analysis. Cells were excluded if input resistance changed $20 \%$ over the entire experiment. Whole cell recordings were conducted by using Multiclamp 700B amplifier (Axon Instruments). All data were collected with $2 \mathrm{kHZ}$ Bessel filter at a $10 \mathrm{kHz}$ sampling frequency (DigiDATA 1550A, Axon Insteuments), and analyzed by Clampfit 10.0 software (Axon Instruments) following low-pass filtering at $1000 \mathrm{~Hz}$. The synaptic response 
belonging to the inhibitory and excitatory amplitude was used to determine the $\mathrm{E} / \mathrm{I}$ ratio (mEPSC amplitude/mIPSC amplitude) of the recorded pyramidal neuron.

\section{Western blot}

PFC samples ( $n=6 /$ group) were harvested for western blot analysis and dissociated in lysis buffer (containing protease inhibitors, $50 \mathrm{mM}$ Tris- $\mathrm{HCl}, \mathrm{pH}$ 7.6) on ice for $30 \mathrm{~min}$ and homogenized via ultrasonification (Ningbo scientz biotechnology CO. LTD, Ningbo, China). After centrifugation at 12,000 g for 10 min at $4{ }^{\circ} \mathrm{C}$, supernatants were collected and protein concentrations were measured with a BCA assay kit (Beyotime Institute of Biotechnology, China). Equivalent amounts of protein samples mixed with gel loading buffer (50 mM Tris-HCl, 10\% SDS, 10\% glycerol, 10\% 2-mercaptoethanol, 2 mg/ml bromophenol blue) were boiled for $5 \mathrm{~min}$ and loaded to SDS-PAGE gels. The separated proteins were electrophoretically transferred to polyvinylidene difluoride membranes. Then the membranes were incubated in blocking buffer ( $5 \%$ fat-free milk in Tween20) for $2 \mathrm{hrs}$ at room temperature and probed with primary antibodies: Rabbit anti-NR2A(1:1000, Cat.No.MAB1555P, Millipore, USA), Rabbit anti-NR2B (1:1000, Cat.No.MAB1557P, Millipore, USA), Rabbit anti-GABA $A_{A}$ a1 (1:1000, Cat.No.224203, Synaptic Systems, Germany), Rabbit anti-GABA $A_{A} \beta 2$ (1:1000, Cat.No.224803, Synaptic Systems, Germany), Mouse anti-SY38 (1:1000, Cat.No.Ab8049, Abcam, UK), Mouse anti-PSD 95 (1:1000, Cat.No.MAB1596, Millipore, USA), Rabbit anti-ErbB4 (1:1000, Cat.No.05-1133, Millipore, USA), Mouse anti-NRG1 (1:1000, Cat.No.sc393006, Santa Cruz Biotechnology, USA) Rabbit anti-GAPDH (1:5000, Cat.No.5174, Cell Signaling Technology, USA) and Rabbit anti- $\beta$-Tubulin (1:5000, Cat.No.2128, Cell Signaling Technology, USA) overnight at $4{ }^{\circ} \mathrm{C}$. After washing in TBST for three times, the membranes were incubated with horseradish peroxidase (HRP) conjugated secondary antibodies diluted 1:5000 (Goat anti-Rabbit (1:5000, Cat.No.Ab6721, Abcam, UK) or Rabbit anti-Mouse(1:5000, Cat.No.Ab6728, Abcam, UK) for 2 hrs at room temperature. The antigen-antibody complexes were detected by enhanced chemiluminescence system (Bio-rad) and visualized by a computer image system (GENE GNOME Chemiluminescence apparatus, Quantity one, Bio-Rad Laboratories). Image processing and semiquantification were performed with Image $\mathrm{J}$ software. Measurements were repeated independently at least 3 times for each experiment. Density of each band was normalized to the internal controls ( $\beta$-tubulin or GAPDH).

\section{Histology and immunohistochemistry}

After antigen recovery, coronal paraffin sections ( $5 \mu \mathrm{m}$ in thickness) were incubated with primary antibodies: mouse anti-parvalbumin (PV, 1:500, Millipore, Cat.No.MAB1572), or anti-GAD67 (1:1000, Millipore, Cat.No.MAB5406) at $4{ }^{\circ} \mathrm{C}$. Signal was detected with Alexafluor 546-labeled fluorescent secondary antibodies (1:1000, Invitrogen, Carlsbad, CA, USA) for GAD67 positive cells, while signal for PV positive cells was detected with a mouse-rabbit ABC kit (PK-6200, Universal, Vector). Images were captured using camera system (SP8 and DMi8 DFC7000J, Leica, Germany). GAD67 and PV positive cells were calculated using Image $\mathrm{J}(\mathrm{NIH}, \mathrm{USA})$ and expressed as cell numbers $/ \mathrm{mm}^{2}$.

\section{Statistical analyses}


All data were presented as mean \pm SEM, and statistical analyses were done with SPSS (SPSS Inc., Chicago, IL, USA). Treatment effects were statistically analyzed by one-way ANOVA followed by Bonferroni's post hoc tests for comparison when normality (and homogeneity of variance) assumptions are satisfied, otherwise Kruskal-Wallis test was applied to analyze the differences between groups followed by Dunn's multiple comparisons tests. Body weight was analyzed using a two-way ANOVA followed by Bonferroni's post hoc tests. A $p$ value $<0.05$ was considered to be of statistical significance.

\section{2) An open-labelled single arm trial Patients' recruitment}

After the completion of pre-clinical experiments, we went to evaluate whether the therapy is effective in patients. An open-labelled single arm, proof-of-concept clinical trial was conducted (registered in ..) from December 24, 2019, to January 20, 2020. After obtaining approval from Ethic committee of and written informed consent from patient or family member, 10 schizophrenia patients (7 men and 3 women; age 18-65 years), who were taking antipsychotics and experiencing an acute exacerbation of psychosis and met all the inclusion criteria (appendix A: Table 1), were recruited (appendix B: Flowchart).

\section{Clinical endpoints and outcome measures}

The primary endpoint was the percentage of the early response at week 2 , and the clinical response was defined as a minimum 30\% reduction in Positive and Negative Syndrome Scale (PANSS) total score. The percent change of PANSS total score was defined as a change /(baseline - 30) x100\% ${ }^{48}$. The secondary endpoints were the change of Brief Psychiatric Rating Scale (BPRS-18) from the baseline to week 1 and week 2 , and the early response rate at week 1 . Safety and tolerability assessments included treatmentemergent adverse events (TEAEs), clinical laboratory tests, electrocardiograms and physiologic measures.

\section{Trial protocol}

Recruited participants received routine antipsychotic medications (in line with standard clinical guidelines; one or two antipsychotics of risperidone, paliperidone, aripiprazole and olanzapine). After fasting for 8hrs and establishing intravenous line, dexmedetomidine, a sedative, was intravenously administrated via an infusion pump with a loading dose of $0.5 \mu \mathrm{g} / \mathrm{kg}$ for $15 \mathrm{~min}$ and then maintained at the rate of $0.1-0.3 \mu \mathrm{g} / \mathrm{kg} / \mathrm{h}$. Sevoflurane was delivered at $2.5 \mathrm{~L} / \mathrm{min}$ in $100 \%$ oxygen for $5 \mathrm{hrs}$ using a face mask under spontaneously respiration. Sevoflurane concentration was started with $6.0 \%$ for first 5-min duration to induce a quick sedation, and then gradually decreased to $0.5-1.5 \%$ and adjusted to maintain the deep sedation. Participants were closely monitored with electrocardiography, pulse oximetry, noninvasive blood pressure, bispectral index (BIS), end-tidal partial pressure of carbon dioxide and 
temperature throughout. This treatment was repeated for 6 times with intervals of $1-2$ days for a total of two weeks (see the detailed protocol in the appendix C).

\section{Statistical Analysis}

Simon's two stage design was used in this proof of concept study. Based on findings of an earlier study, the percentage of patients with at least $30 \%$ improvement in total PANSS at 2 weeks was about $30 \% 49$. In this study, a 70\% or higher early response rate was set as the target of "good" and 30\% was set as "poor". The optimal two-stage design to test the null hypothesis that $\mathrm{P}<=0.300$ versus the alternative that $\mathrm{P}>=$ 0.700 has an expected sample size of 6.08 . After testing the drug on 2 patients in the first stage, the trial was planned to be terminated if 0 respond. If the trial goes on to the second stage, a total of 10 patients was planned to be studied. If the total number of patients responding is less than or equal to 5 , the drug was rejected.

The efficacy and safety analysis were conducted in the full analysis set, which included all patients who received at least one treatment of sevoflurane inhalation and had at least one evaluation post-baseline. The early response of BPRS-18 total score at week 1 and week 2 was reported as mean with standard deviation.

\section{Declarations}

\section{Acknowledgements}

\section{Author contributions}

\section{Funding:}

\section{Conflict of interest}

The authors declare that they have no conflict of interest.

\section{References}


1. Simeone JC, Ward AJ, Rotella P, Collins J, Windisch R. An evaluation of variation in published estimates of schizophrenia prevalence from 1990 horizontal line 2013: a systematic literature review. BMC Psychiatry 15, 193 (2015).

2. Global Burden of Disease Study C. Global, regional, and national incidence, prevalence, and years lived with disability for 301 acute and chronic diseases and injuries in 188 countries, 1990-2013: a systematic analysis for the Global Burden of Disease Study 2013. Lancet 386, 743-800 (2015).

3. Stahl SM. Beyond the dopamine hypothesis of schizophrenia to three neural networks of psychosis: dopamine, serotonin, and glutamate. CNS Spectr 23, 187-191 (2018).

4. Carbon $\mathrm{M}$, Correll $\mathrm{CU}$. Thinking and acting beyond the positive: the role of the cognitive and negative symptoms in schizophrenia. CNS Spectr 19 Suppl 1, 38-52; quiz 35-37, 53 (2014).

5. Correll CU, Rubio JM, Inczedy-Farkas G, Birnbaum ML, Kane JM, Leucht S. Efficacy of 42 Pharmacologic Cotreatment Strategies Added to Antipsychotic Monotherapy in Schizophrenia: Systematic Overview and Quality Appraisal of the Meta-analytic Evidence. JAMA Psychiatry 74, 675-684 (2017).

6. Lieberman JA. Effectiveness of antipsychotic drugs in patients with chronic schizophrenia: efficacy, safety and cost outcomes of CATIE and other trials. J Clin Psychiatry 68, e04 (2007).

7. Millan MJ, Fone K, Steckler T, Horan WP. Negative symptoms of schizophrenia: clinical characteristics, pathophysiological substrates, experimental models and prospects for improved treatment. Eur Neuropsychopharmacol 24, 645-692 (2014).

8. In: 2019 exceptional surveillance of psychosis and schizophrenia in adults: prevention and management (NICE guideline CG178)) (2019).

9. Martinez-Raga J, et al. 1st International Experts' Meeting on Agitation: Conclusions Regarding the Current and Ideal Management Paradigm of Agitation. Front Psychiatry 9, 54 (2018).

10. Sieghart W. Allosteric modulation of GABAA receptors via multiple drug-binding sites. Adv Pharmacol 72, 53-96 (2015).

11. Rudolph U, Knoflach F. Beyond classical benzodiazepines: novel therapeutic potential of GABAA receptor subtypes. Nat Rev Drug Discov 10, 685-697 (2011).

12. Khayenko V, Maric HM. Targeting GABAAR-Associated Proteins: New Modulators, Labels and Concepts. Front Mol Neurosci 12, 162 (2019).

13. Rudolph U, Antkowiak B. Molecular and neuronal substrates for general anaesthetics. Nat Rev Neurosci 5, 709-720 (2004).

14. Jerath A, et al. Safety and Efficacy of Volatile Anesthetic Agents Compared With Standard Intravenous Midazolam/Propofol Sedation in Ventilated Critical Care Patients: A Meta-analysis and Systematic Review of Prospective Trials. Anesth Analg 124, 1190-1199 (2017).

15. Olsen RW, Sieghart W. GABA A receptors: subtypes provide diversity of function and pharmacology. Neuropharmacology 56, 141-148 (2009). 
16. Lorenz-Guertin JM, Jacob TC. GABA type a receptor trafficking and the architecture of synaptic inhibition. Dev Neurobiol 78, 238-270 (2018).

17. Brohan J, Goudra BG. The Role of GABA Receptor Agonists in Anesthesia and Sedation. CNS Drugs 31, 845-856 (2017).

18. McCartney MR, Deeb TZ, Henderson TN, Hales TG. Tonically active GABAA receptors in hippocampal pyramidal neurons exhibit constitutive GABA-independent gating. Mol Pharmacol 71, 539-548 (2007).

19. Chkhartishvili E, Maglakelidze N, Babilodze M, Chijavadze E, Nachkebia N. Changes of open field behavior in animal model of depression. Georgian Med News 11, 107-112 (2011).

20. Castanheira L, Ferreira MF, Sebastiao AM, Telles-Correia D. Anxiety Assessment in Pre-clinical Tests and in Clinical Trials: A Critical Review. Curr Top Med Chem 18, 1656-1676 (2018).

21. Sankoorikal GM, Kaercher KA, Boon CJ, Lee JK, Brodkin ES. A mouse model system for genetic analysis of sociability: C57BL/6J versus BALB/cJ inbred mouse strains. Biol Psychiatry 59, 415-423 (2006).

22. Akillioglu K, Binokay S, Kocahan S. The effect of neonatal N-methyl-D-aspartate receptor blockade on exploratory and anxiety-like behaviors in adult BALB/C and C57BL/6 mice. Behav Brain Res 233, 157-161 (2012).

23. Lim AL, Taylor DA, Malone DT. Consequences of early life MK-801 administration: Iong-term behavioural effects and relevance to schizophrenia research. Behav Brain Res 227, 276-286 (2012).

24. Kocahan S, Akillioglu K, Binokay S, Sencar L, Polat S. The effects of N-Methyl-D-Aspartate receptor blockade during the early neurodevelopmental period on emotional behaviors and cognitive functions of adolescent Wistar rats. Neurochem Res 38, 989-996 (2013).

25. Krystal JH, et al. Subanesthetic effects of the noncompetitive NMDA antagonist, ketamine, in humans. Psychotomimetic, perceptual, cognitive, and neuroendocrine responses. Arch Gen Psychiatry 51, 199-214 (1994).

26. Lahti AC, Koffel B, LaPorte D, Tamminga CA. Subanesthetic doses of ketamine stimulate psychosis in schizophrenia. Neuropsychopharmacology 13, 9-19 (1995).

27. Bubenikova-Valesova V, Horacek J, Vrajova M, Hoschl C. Models of schizophrenia in humans and animals based on inhibition of NMDA receptors. Neurosci Biobehav Rev 32, 1014-1023 (2008).

28. Sanz-Clemente A, Nicoll RA, Roche KW. Diversity in NMDA receptor composition: many regulators, many consequences. Neuroscientist 19, 62-75 (2013).

29. Paoletti P, Bellone C, Zhou Q. NMDA receptor subunit diversity: impact on receptor properties, synaptic plasticity and disease. Nat Rev Neurosci 14, 383-400 (2013).

30. Lujan R, Shigemoto R, Lopez-Bendito G. Glutamate and GABA receptor signalling in the developing brain. Neuroscience 130, 567-580 (2005).

31. Lee G, Zhou Y. NMDAR Hypofunction Animal Models of Schizophrenia. Front Mol Neurosci 12, 185 (2019). 
32. Yamamoto H, Hagino Y, Kasai S, Ikeda K. Specific Roles of NMDA Receptor Subunits in Mental Disorders. Curr Mol Med 15, 193-205 (2015).

33. Xi D, Zhang W, Wang HX, Stradtman GG, Gao WJ. Dizocilpine (MK-801) induces distinct changes of $\mathrm{N}$-methyl-D-aspartic acid receptor subunits in parvalbumin-containing interneurons in young adult rat prefrontal cortex. Int J Neuropsychopharmacol 12, 1395-1408 (2009).

34. Wilson MA, Kinsman SL, Johnston MV. Expression of NMDA receptor subunit mRNA after MK-801 treatment in neonatal rats. Brain Res Dev Brain Res 109, 211-220 (1998).

35. Chung DW, Volk DW, Arion D, Zhang Y, Sampson AR, Lewis DA. Dysregulated ErbB4 Splicing in Schizophrenia: Selective Effects on Parvalbumin Expression. Am J Psychiatry 173, 60-68 (2016).

36. Geddes AE, Huang XF, Newell KA. Reciprocal signalling between NR2 subunits of the NMDA receptor and neuregulin1 and their role in schizophrenia. Prog Neuropsychopharmacol Biol Psychiatry 35, 896-904 (2011).

37. Packer AM, Yuste R. Dense, unspecific connectivity of neocortical parvalbumin-positive interneurons: a canonical microcircuit for inhibition? J Neurosci 31, 13260-13271 (2011).

38. Ferguson BR, Gao WJ. PV Interneurons: Critical Regulators of E/I Balance for Prefrontal CortexDependent Behavior and Psychiatric Disorders. Front Neural Circuits 12, 37 (2018).

39. Kolbitsch C, et al. A subanesthetic concentration of sevoflurane increases regional cerebral blood flow and regional cerebral blood volume and decreases regional mean transit time and regional cerebrovascular resistance in volunteers. Anesth Analg 91, 156-162 (2000).

40. Chen C, et al. Low-dose sevoflurane promotes hippocampal neurogenesis and facilitates the development of dentate gyrus-dependent learning in neonatal rats. ASN Neuro 7, (2015).

41. Chen X, et al. Neonatal Exposure to Low-Dose (1.2\%) Sevoflurane Increases Rats' Hippocampal Neurogenesis and Synaptic Plasticity in Later Life. Neurotox Res 34, 188-197 (2018).

42. Hernandez-Vazquez F, Garduno J, Hernandez-Lopez S. GABAergic modulation of serotonergic neurons in the dorsal raphe nucleus. Rev Neurosci 30, 289-303 (2019).

43. Zhu D, et al. Postnatal Administration of Dizocilpine Inhibits Neuronal Excitability in PFC and Induces Social Deficits Detected by MiceProfiler. Mol Neurobiol 54, 8152-8161 (2017).

44. Murueta-Goyena A, Ortuzar N, Gargiulo PA, Lafuente JV, Bengoetxea H. Short-Term Exposure to Enriched Environment in Adult Rats Restores MK-801-Induced Cognitive Deficits and GABAergic Interneuron Immunoreactivity Loss. Mol Neurobiol 55, 26-41 (2018).

45. Batinic B. Cognitive Models of Positive and Negative Symptoms of Schizophrenia and Implications for Treatment. Psychiatr Danub 31, 181-184 (2019).

46. Silverman JL, Yang M, Lord C, Crawley JN. Behavioural phenotyping assays for mouse models of autism. Nature reviews Neuroscience 11, 490-502 (2010).

47. Liang J, Xu W, Hsu YT, Yee AX, Chen L, Sudhof TC. Conditional neuroligin-2 knockout in adult medial prefrontal cortex links chronic changes in synaptic inhibition to cognitive impairments. Molecular psychiatry 20, 850-859 (2015). 
48. Leucht S, Davis JM, Engel RR, Kissling W, Kane JM. Definitions of response and remission in schizophrenia: recommendations for their use and their presentation. Acta Psychiatr Scand Suppl, 714 (2009).

49. Kinon BJ, et al. Early response to antipsychotic drug therapy as a clinical marker of subsequent response in the treatment of schizophrenia. Neuropsychopharmacology 35, 581-590 (2010).

\section{Figures}

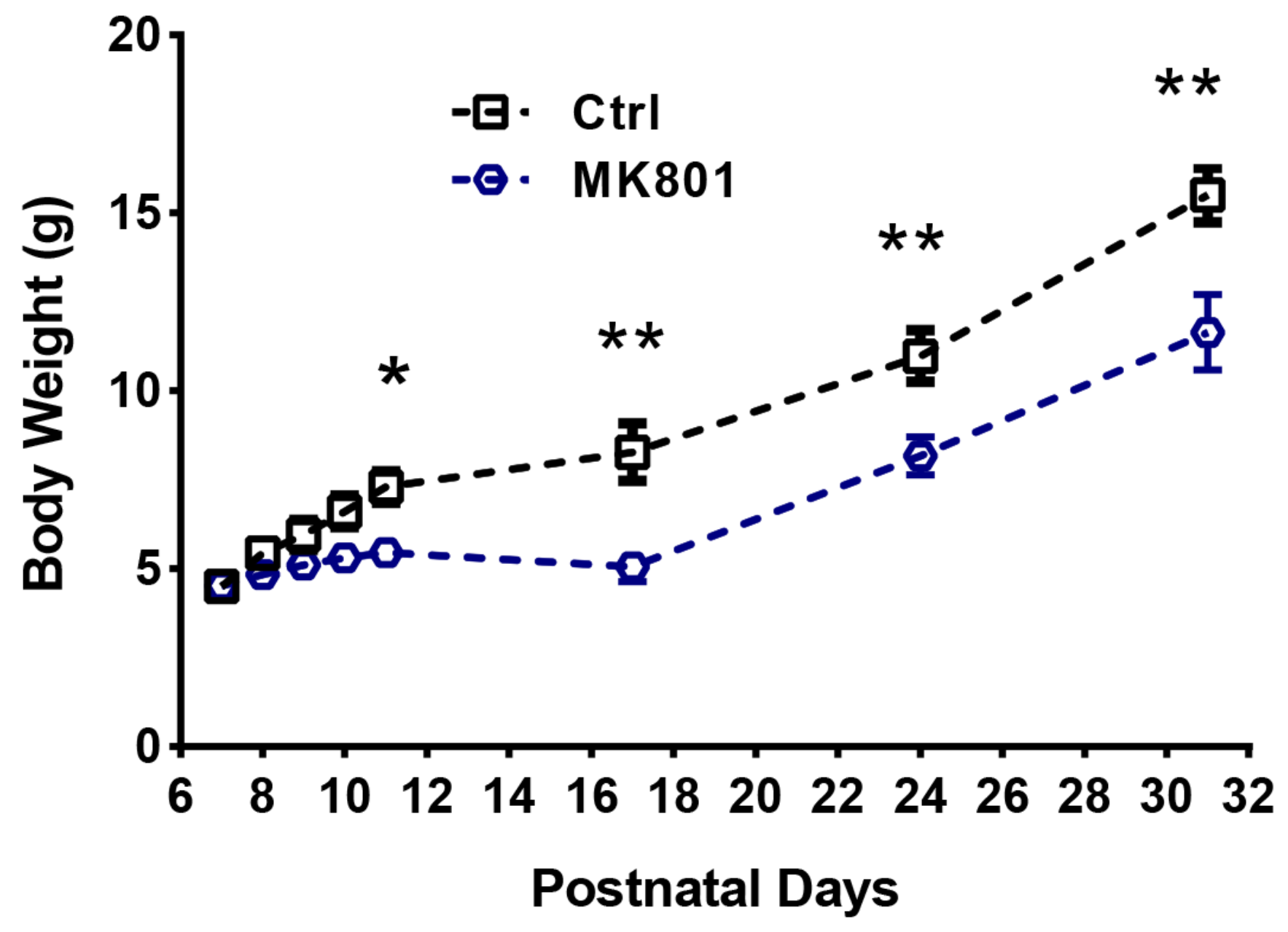

Figure 1

Effect of MK801 administration during neonatal period on body weight The body weight of the MK801 group was lower after 5-day systemic administration of MK801 (0.5 mg/kg, i.p., twice/day) compared with control group, and this difference persisted thereafter. Data are analysed by two-way repeated measures ANOVA followed by Bonferroni's post-hoc test. Data are represented as mean \pm SEM $(n=18)$; ${ }^{*} \mathrm{p}<0.05 ; * \star \mathrm{p}<0.01, * \star \star \mathrm{p}<0.001$. 
Ctrl

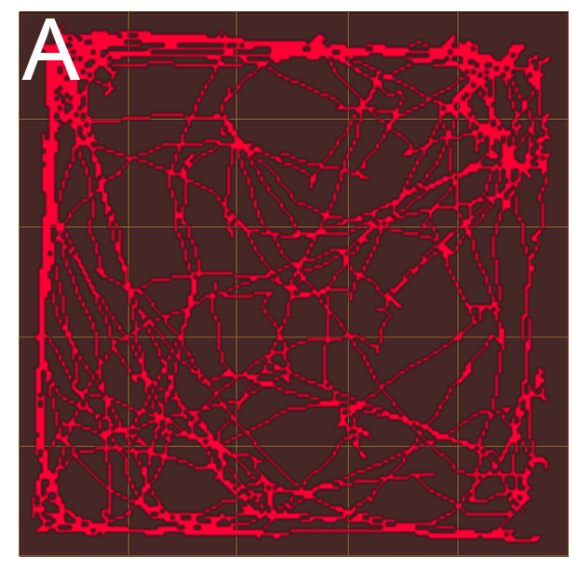

D
MK801
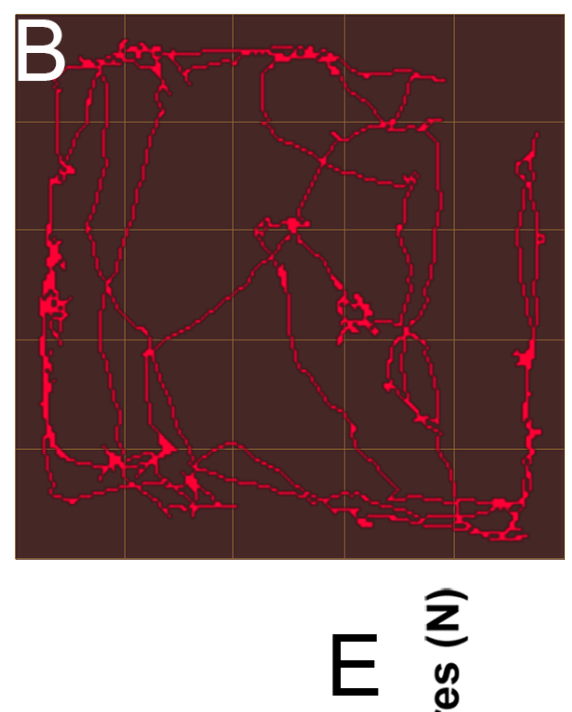
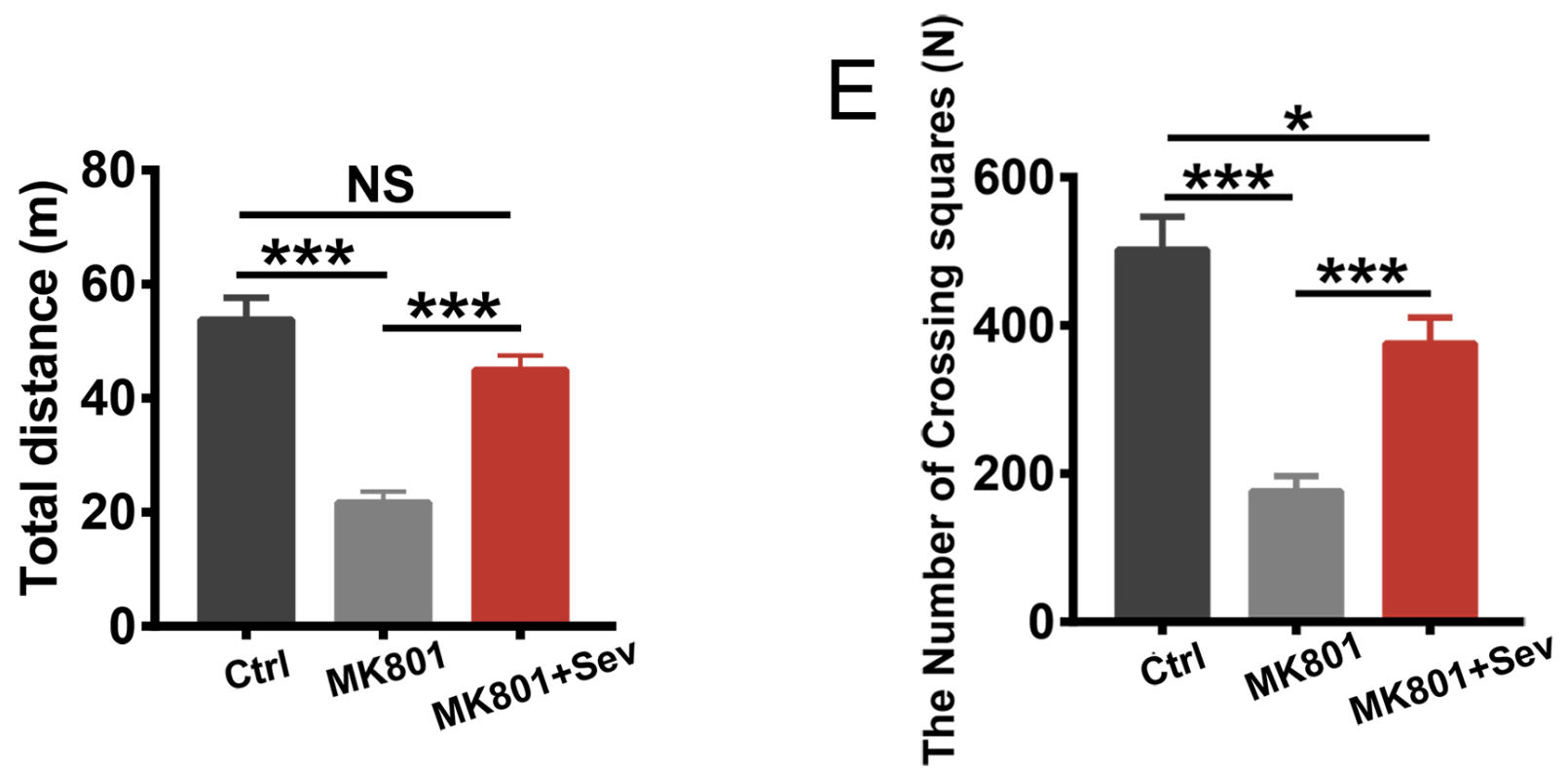

Figure 2

Sevoflurane (1\%) for $1 \mathrm{hr}$ for five consecutive days protected against MK801-induced hypoactivity in the open field test (OFT) The recording trail trace from a mouse in the control (A), MK801 (B) and MK801+Sev group (C); (D) Statistics showed a significant difference in total distance travelled in the OFT among three groups. (E) Statistics showed a significant difference of the frequency of crossing squares in the OFT among three groups. Data are analysed by one-way ANOVA followed by Bonferroni's post-hoc test. Data are represented as mean \pm SEM $(n=18) ;{ }^{*} p<0.05 ; * \star p<0.01, * \star * p<0.001$. 
A
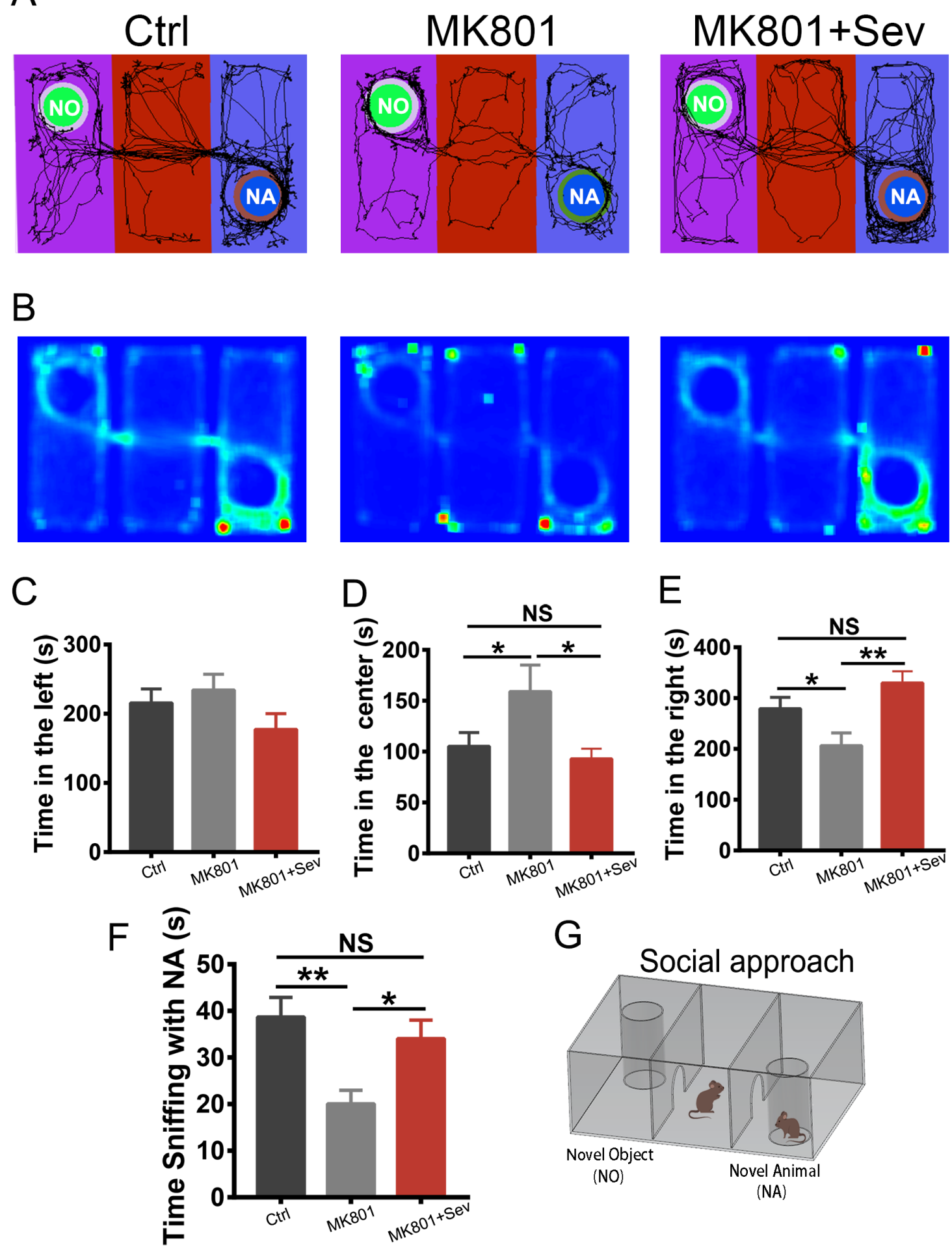

\section{Figure 3}

Sevoflurane (1\%) for $1 \mathrm{hr}$ for consecutive 5 days protected against MK801-induced social interaction defects (A) Examples of recording trails from control (left), MK801 (middle), and MK801+Sev group (right), respectively. (B) Examples of recording density map from control (left), MK801(middle), and MK801+Sev group (right), respectively. (C) Quantification of time spent in the left chamber showed no significant difference of among three groups. (D) Statistics showed a significant difference of time spent 
in the middle chamber among three groups. (E) Statistics showed a significant difference of time spent in the right chamber among three groups. (F) Statistics showed a significant difference of time sniffing NA (novel animal). (G) Schematic of three-chambered apparatus. One-way ANOVA followed by Bonferroni's post-hoc tests was used for analysis. Data are represented as mean $\pm \operatorname{SEM}(n=18) ;{ }^{\star} p<0.05 ; * \star p<0.01$, $\star \star * \mathrm{p}<0.001$.

A

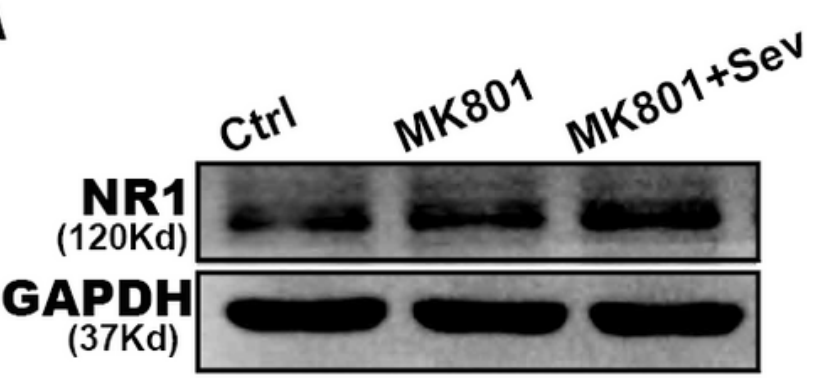

B

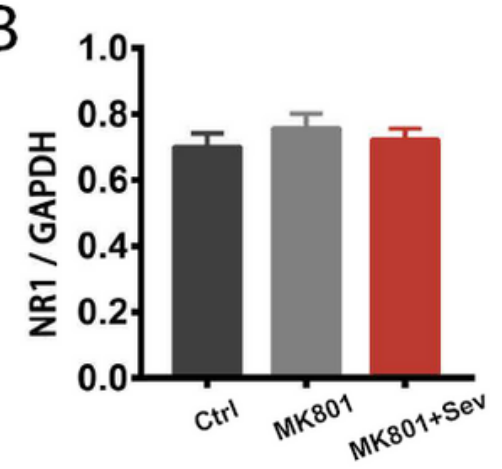

C

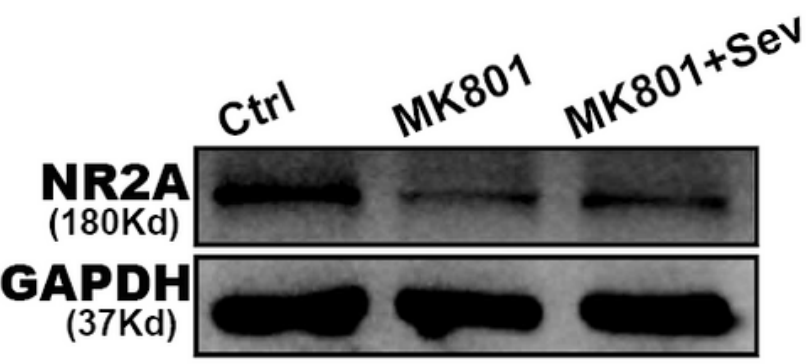

E

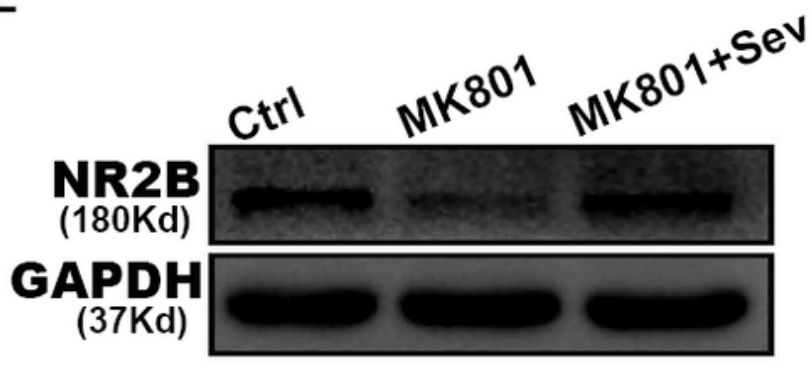

G

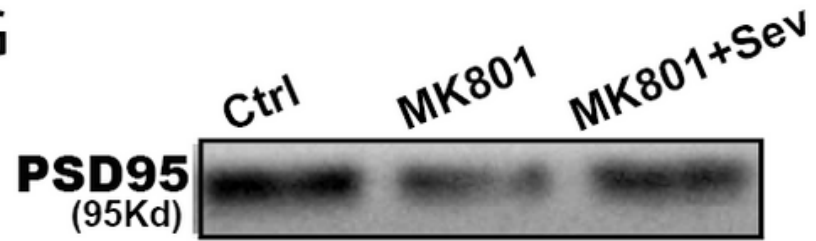

\section{及-Tubulin \\ $(55 \mathrm{Kd})$}

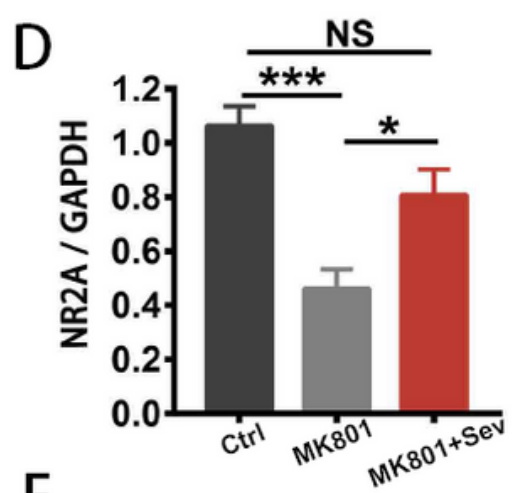

$\mathrm{F}$
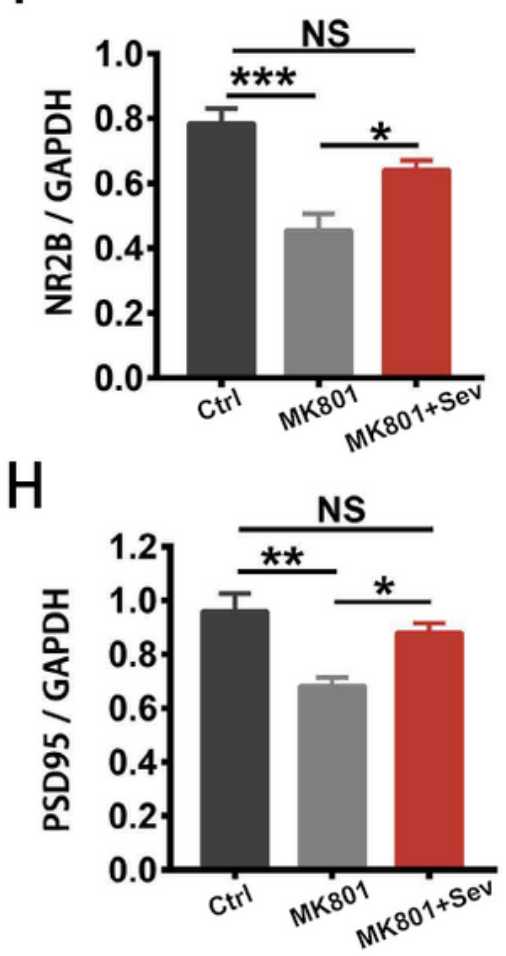

Figure 4 
Sevoflurane (1\%) for $1 \mathrm{hr}$ for consecutive 5 days reversed MK801-induced aberrant NMDARs composition in the mPFC (A) Representative bands of NMDAR subunit NR1 expression in the mPFC. (B) Quantification data comparing NR1 band intensity among three groups. (C) Representative bands of NMDAR subunit NR2A expression in the mPFC. (D) T Quantification data comparing NR2A band intensity among three groups. (E) Reprehensive bands of NMDAR subunit NR2B expression in the mPFC. (F) Quantification data comparing NR2B band intensity among three groups. (G) Representative bands of PSD95 expression in the mPFC. (H) Quantification data comparing PSD95 band intensity among three groups. One-way ANOVA followed by Bonferroni's post-hoc tests was used for analysis. Data are represented as mean \pm $\operatorname{SEM}(n=6) ;{ }^{*}<0.05 ; * \star p<0.01, * \star \star p<0.001$.

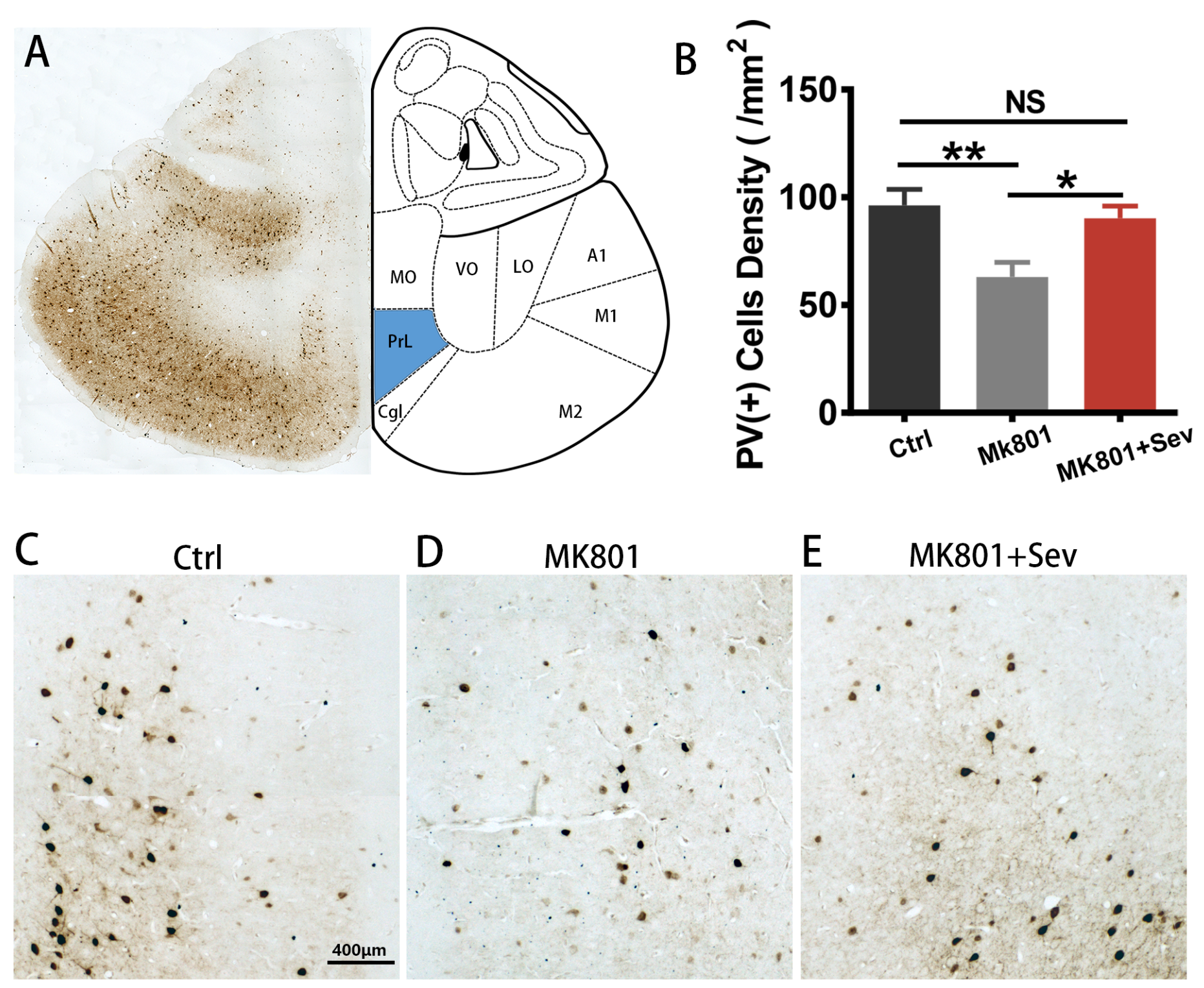

\section{Figure 5}

Sevoflurane (1\%) for $1 \mathrm{hr}$ for consecutive 5 days rescued MK801-induced reduction of PV positive interneurons in the mPFC. (A) Schematic illustration indicates the mPFC (blue area) in the prefrontal 
cortex where images were taken and analysis. (B) Statistic analysis showed a significant decrease of PVpositive interneurons in MK801 group compared to the control (Ctrl), and after sevoflurane inhalation treatment, PV-positive interneurons were significantly increased compared to the MK801 group. (C-E) Representative images of PV-positive interneurons taken from laminae II/III of the MPFC in the control (left), MK801 (middle) and MK801+Sev (right) group, respectively. One-way ANOVA followed by Bonferroni's post-hoc tests was used for analysis. Data are represented as mean $\pm \operatorname{SEM}(n=6) ;{ }^{*} p<0.05$; $\star \star p<0.01, * \star \star p p<0.001$.

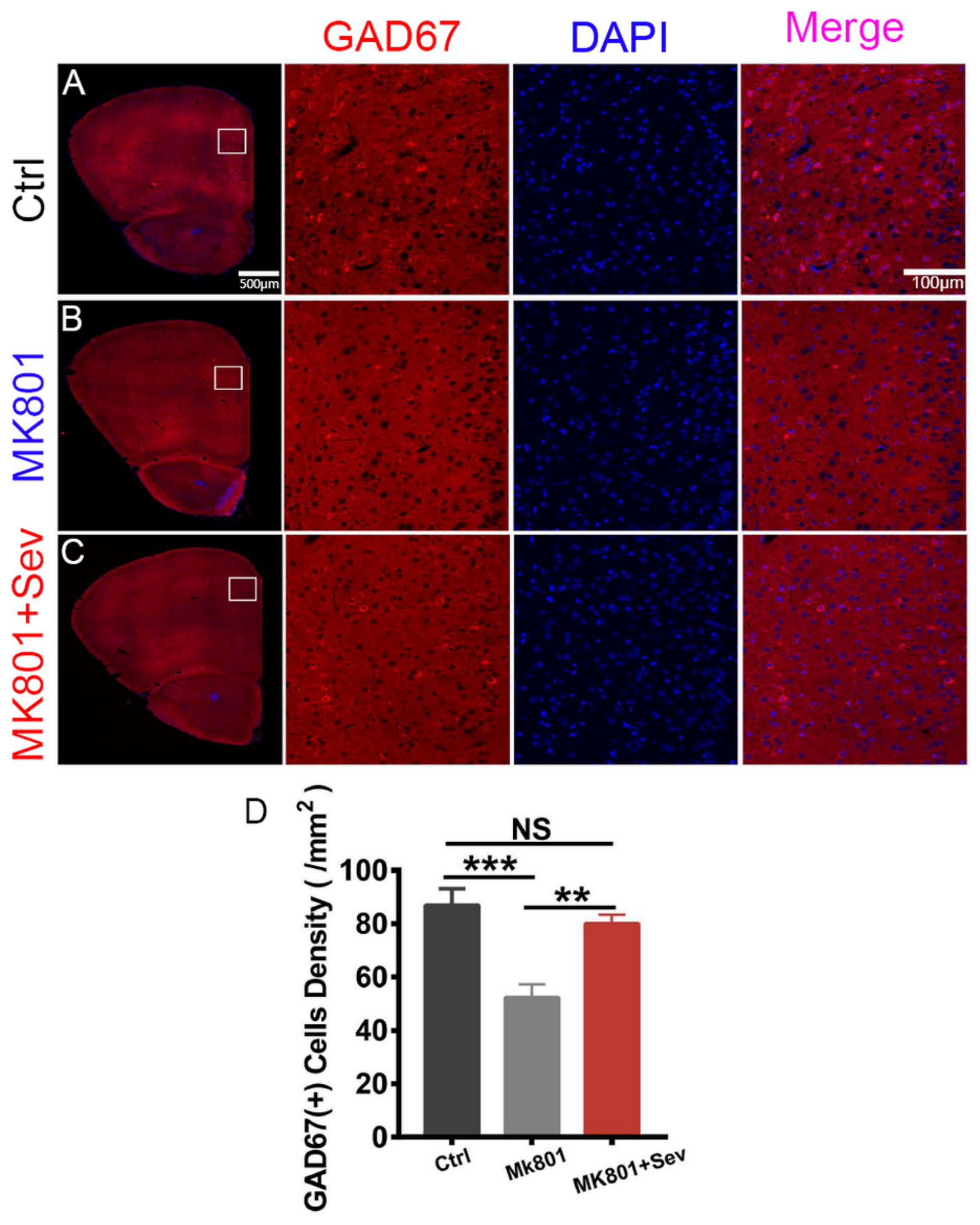




\section{Figure 6}

Sevoflurane (1\%) for $1 \mathrm{hr}$ for consecutive 5 days rescued MK801-induced reduction of GAD 67 positive interneurons in the mPFC. (A-C) The left panel represents low-resolution image of anti-GAD67 immunofluorescence staining of the control (A), MK801 (B) and MK801+Sev (C), with boxed area showing high resolution image of anti-GAD67 immunofluorescence staining and DAPI taken for analysis. (D) Statistic analysis showed a significant decrease in GAD67- positive interneurons in MK801 group compared to the control (Ctrl), and after sevoflurane inhalation treatment, GAD67-positive interneurons were significantly increased compared to the MK801 group. One-way ANOVA followed by Bonferroni's post-hoc tests was used for analysis. Data are represented as mean $\pm \operatorname{SEM}(n=7) ;{ }^{\star} p<0.05 ; * \star p<0.01$, $\star \star \star p<0.001$. 
A

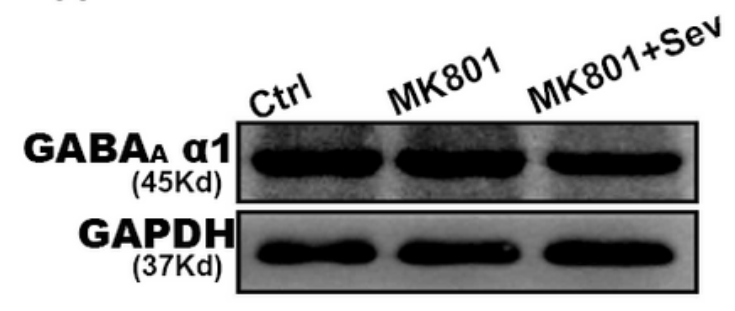

C

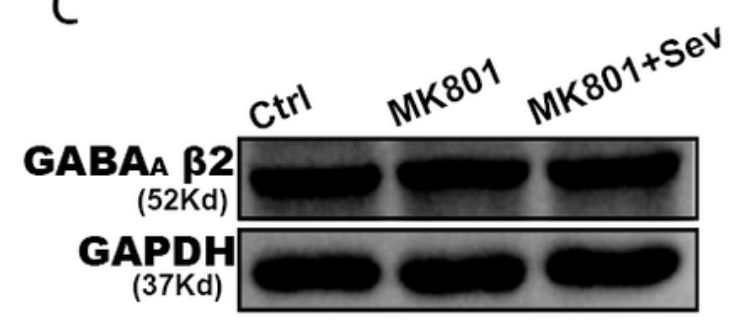

B

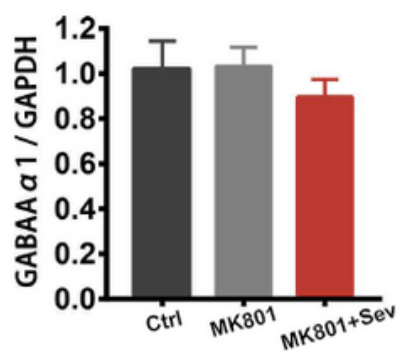

D
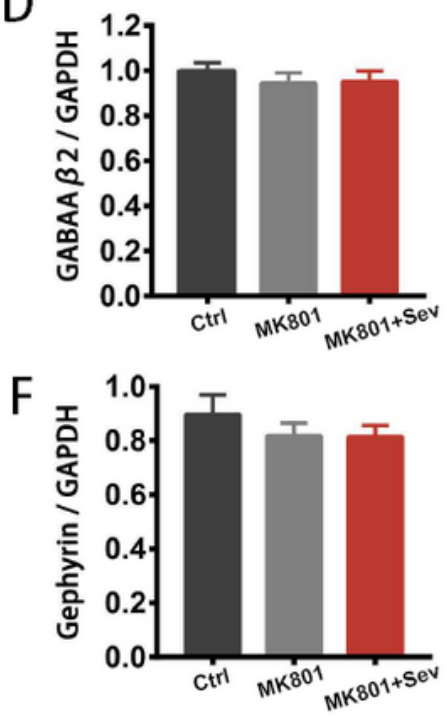

G

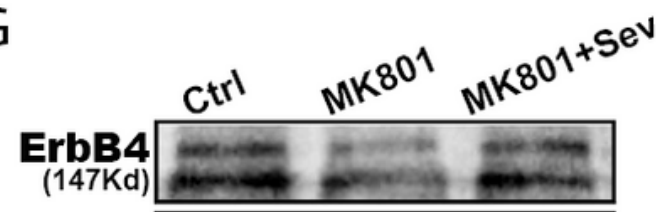

GAPDH (37Kd)
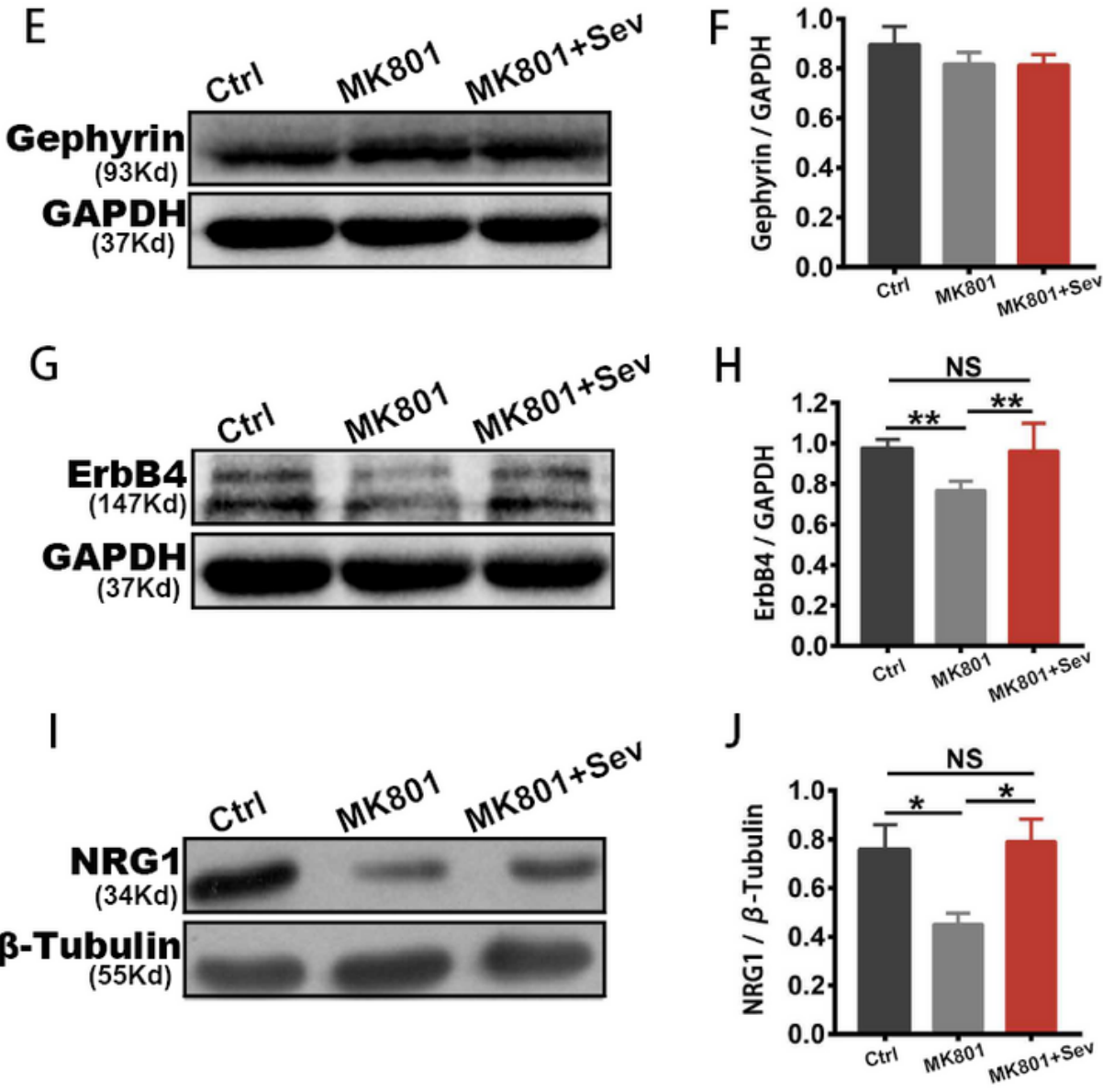

Figure 7

Sevoflurane (1\%) for $1 \mathrm{hr}$ for consecutive 5 days reversed MK801-induced alterations to ErbB4-NRG1 signal pathway in the mPFC. (A) Representative band of GABAAR subunit a1 expression in the mPFC. (B) Quantification of band intensity of GABAAR subunit a1 among three groups. (C) Representative band of GABAAR subunit $\beta 2$ expression in the mPFC. (D) Quantification of band intensity of GABAAR subunit $\beta 2$ among three groups. (E) Representative band of GABAAR anchoring protein gephyrin expression in the 
mPFC. (F) Quantification of band intensity of gephyrin among three groups. (G) Representative band of ErbB4 expression in the mPFC. (H) Quantification of band intensity of ErbB4 among three groups. (I) Representative band of NRG1 expression in the mPFC. (J) Quantification of band intensity of NRG1 among three groups. One-way ANOVA followed by Bonferroni's post-hoc tests was used for analysis. Data are represented as mean \pm SEM $(n=6) ;{ }^{*} p<0.05 ; * * p<0.01$, $* * * p<0.001$.

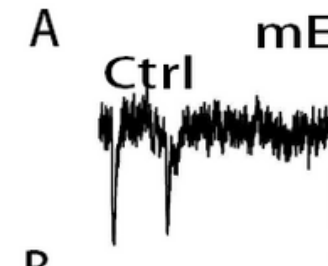

B

MK801

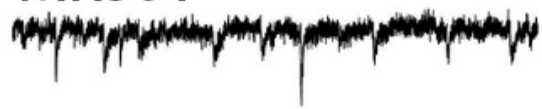

C

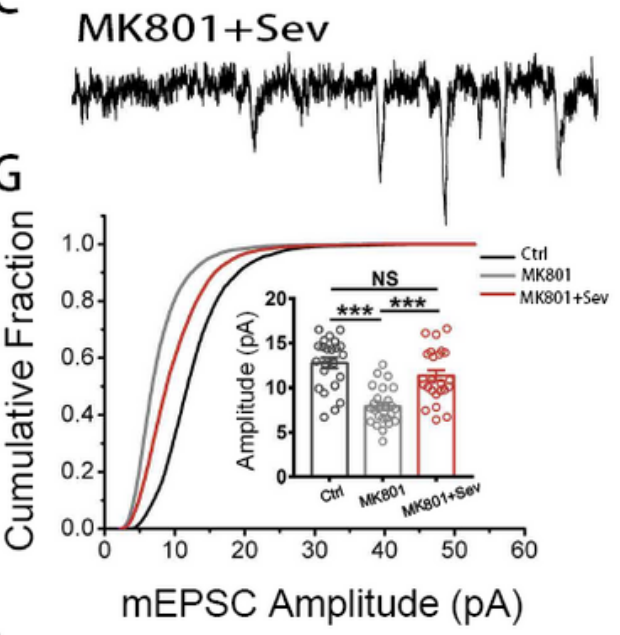

I

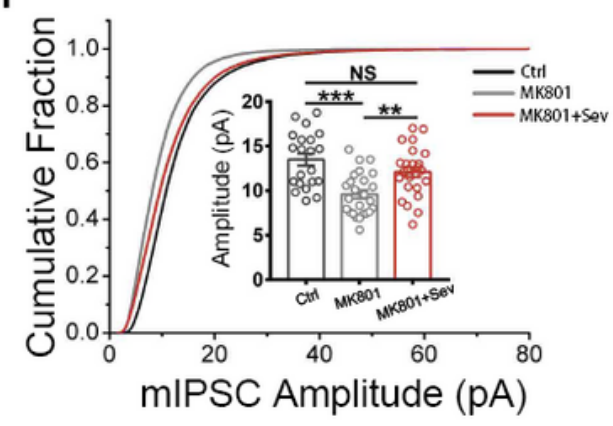

$\mathrm{K}$
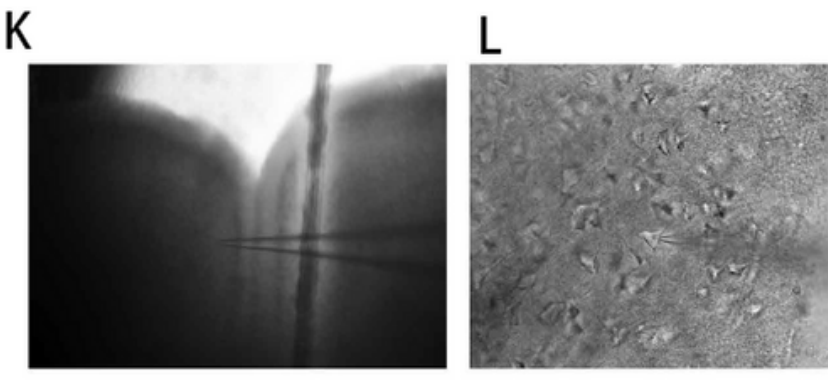

mIPSC

D

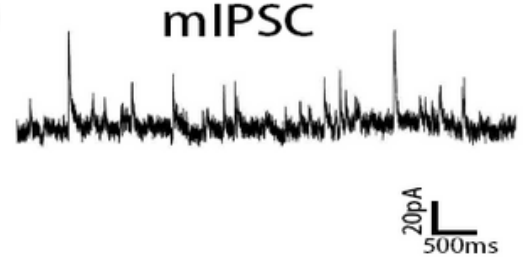

E

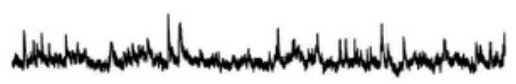

F

$\mathrm{H}$

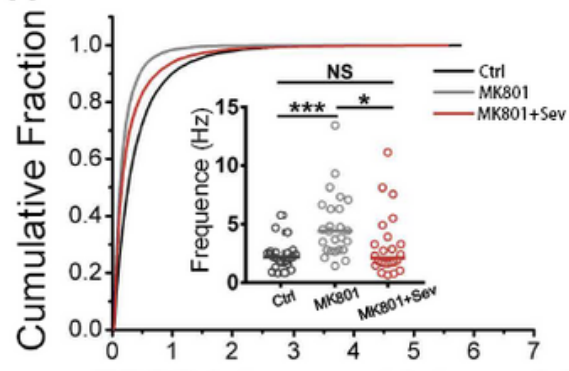

mEPSC Inter-event Interva (s)

J
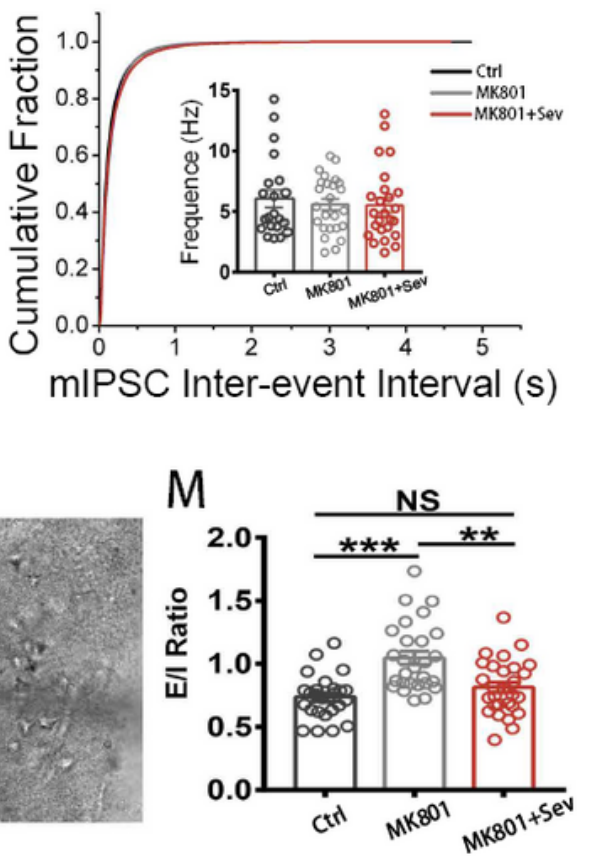

Figure 8 
Sevoflurane (1\%) for $1 \mathrm{hr}$ for consecutive 5 days reversed Mk801-induced alterations to electrophysiological profiles and $\mathrm{E} / \mathrm{I}$ balance in the mPFC. (A-C) Representative mEPSC recording traces of pyramidal neurons in laminae II/III of the mPFC from control (A), MK801(B) and MK801+Sev (C) mice. (D-F) Representative mIPSC recording traces of pyramidal neurons in laminae II/III of the $\mathrm{mPFC}$ from control (D), MK801(E) and MK801+Sev (F) mice. (G) Cumulative distribution of mEPSCs amplitudes recorded from control (black line), MK801 (gray line) and MK801+Sev (red line) mice. Inset, mean amplitude of mEPSCs recorded from layer II/III pyramidal neurons in the PFC of Control (23 cells $/ 5$ mice), MK801 (25 cells/ 5 mice) and MK801+Sev (24 cells/5 mice) group mice. (H) Cumulative distribution of mEPSCs inter-event intervals recorded from control (black line), MK801 (gray line) and MK801+Sev (red line) group mice. Inset, mean frequency of mEPSCs recorded from layer II/III pyramidal neurons in the PFC of Control (23 cells/ 5 mice), MK801(25 cells/5 mice) and MK801+Sev (24 cells/5 mice) mice. (I) Cumulative distribution of mIPSCs amplitudes recorded from control (black line), Mk801 (gray line) and MK801+Sev (red line) mice. Inset, mean amplitude of mIPSCs recorded from layer II/III pyramidal neurons in the PFC of Control (21 cells/5 mice), MK801 (25 cells/5 mice) and MK801+Sev (25cells/5 mice) group mice. $(\mathrm{J})$ Cumulative distribution of mIPSCs inter-event intervals recorded from control (black line), MK801 (gray line) and MK801+Sev (red line) group mice. Inset, mean frequency of mIPSCs recorded from layer II/III pyramidal neurons in the PFC of Control (21cells/ 5 mice), MK801 (25 cells/ 5 mice) and MK801+Sev (25 cells/5 mice) mice. (K, L) Laminar II/III of the mPFC pyramidal neurons viewed under infrared optics were recorded with a patch-clamp recording pipette. (M) The E/I ratio of mEPSC amplitude to mIPSC amplitude in the same pyramidal neuron showed significant increase in the MK801 group compared to the control. In contrast, after 1-hr $1 \%$ sevoflurane inhalation for consecutive 5 days, the E/I ratio was significantly decreased compared to the MK801 mice. (Ctrl: $0.74 \pm 0.03,26$ cells from 5 mice in control group; MK801: $1.05 \pm 0.05,27$ cells from 5 mice; MK801+Sev: $0.81 \pm 0.04,28$ cells from 5 mice). One-way ANOVA followed by Bonferroni's post-hoc tests and Kruskal-Wallis test followed by Dunn's multiple comparison test were used for analysis. Data are represented as mean $\pm \operatorname{SEM}(n=5) ;{ }^{\star} p<0.05$; $\star * \mathrm{p}<0.01, * * * \mathrm{p}<0.001$

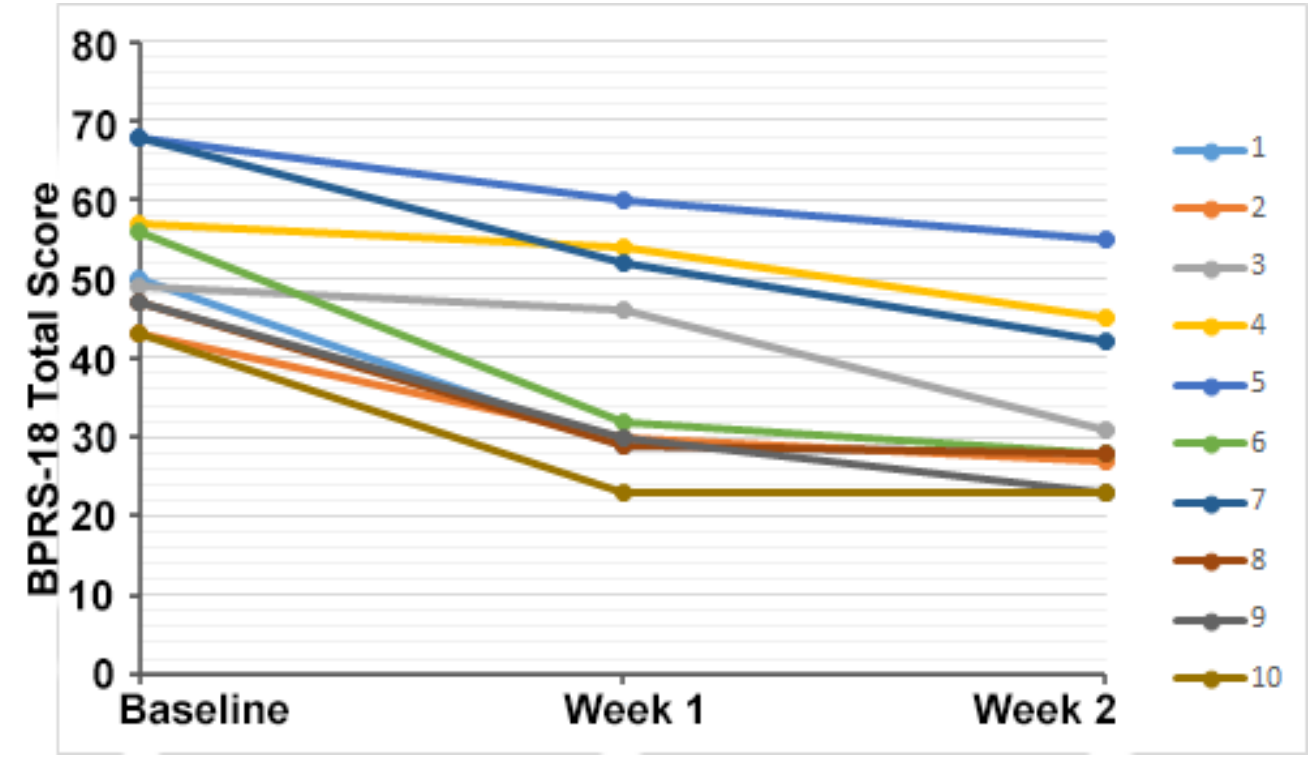

\section{Figure 9}


Low-concentration sevoflurane improved schizophrenia patients' symptoms. Individual 18-item Brief Psychiatric Rating Scale (BPRS-18) total scores were assessed at the week 1 and week 2 after initiation of sevoflurane inhalation.

\section{Supplementary Files}

This is a list of supplementary files associated with this preprint. Click to download.

- Appendicesinonefileblinded.docx 